\title{
LEADING EDGE FLOW STRUCTURE OF A DYNAMICALLY PITCHING NACA 0012 AIRFOIL
}

\author{
A Thesis \\ by \\ BRANDON JAMES PRUSKI \\ Submitted to the Office of Graduate Studies of \\ Texas A\&M University \\ in partial fulfillment of the requirements for the degree of \\ MASTER OF SCIENCE
}

Approved by:

Chair of Committee, Rodney Bowersox

Committee Members, Helen Reed

Simon North

Head of Department, Rodney Bowersox

December 2012

Major Subject: Aerospace Engineering

Copyright 2012 Brandon James Pruski 


\begin{abstract}
The leading edge flow structure of the NACA 0012 airfoil is experimentally investigated under dynamic stall conditions $\left(M=0.1 ; \alpha=16.7^{\circ}, 22.4^{\circ} ; R e_{c}=1 \times 10^{6}\right)$ using planar particle image velocimetry. The airfoil was dynamically pitched about the $1 / 4$ chord at a reduced frequency, $k=0.1$. As expected, on the upstroke the flow remains attached in the leading edge region above the static stall angle, whereas during downstroke, the flow remains separated below the static stall angle. A phase averaging procedure involving triple velocity decomposition in combination with the Hilbert transform enables the entire dynamic stall process to be visualized in phase space, with the added benefit of the complete phase space composed of numerous wing oscillations. The formation and complex evolution of the leading edge vortex is observed. This vortex is seen to grow, interact with surrounding vorticity, detach from the surface, and convect downstream. A statistical analysis coupled with instantaneous realizations results in the modification of the classical dynamic stall conceptual model, specifically related to the dynamics of the leading edge vortex.
\end{abstract}


for my family 


\section{ACKNOWLEDGEMENTS}

The author would like to thank the Army Research Office for sponsoring this study, and acknowledge the Oran W. Nicks low-speed wind-tunnel staff at Texas A\&M University for providing assistance with the installation of the dynamic stall apparatus and operation of the wind tunnel. Additionally, without the help of Dr. R. A. Humble this document and the research that was conducted would have never been possible. Furthermore, I would like to thank the researchers at the National Aerothermochemisty Lab (Michael Semper, Scott Peltier, Chi Mai) and the Hypersonic Research Center (Jerrod Hofferth and Alex Craig) for their insightful comments and diagnostic knowledge that provided the foundation of my research capabilities. Finally I would like to thank Dr. Bowersox for the opportunity and guidance that made this thesis possible. 


\section{TABLE OF CONTENTS}

ABSTRACT ................................ ii

DEDICATION ...................................

ACKNOWLEDGEMENTS .............................. iv

TABLE OF CONTENTS . . . . . . . . . . . . . . . . . . v v

LIST OF FIGURES . . . . . . . . . . . . . . . . . . . . vii vi

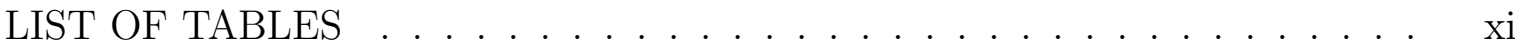

CHAPTER

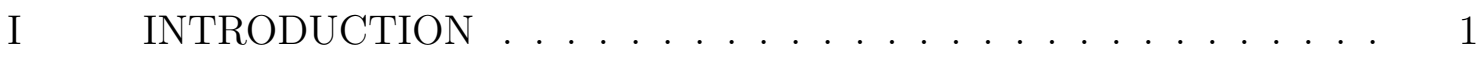

A. Dynamic Stall Overview . . . . . . . . . . . . . . . 1

B. Conceptual Model . . . . . . . . . . . . . . 3

C. Motivation . . . . . . . . . . . . . 6

D. Research Objectives and Scientific Contribution . . . . . 7

E. Previous Work in Texas A\&M DSF . . . . . . . . . 8

F. Thesis Outline . . . . . . . . . . . . . . . 8

II EXPERIMENTAL SETUP . . . . . . . . . . . . . . . 9

A. Facility . . . . . . . . . . . . . . . . 9

B. Experimental Conditions . . . . . . . . . . . . . . 11

III DIAGNOSTICS AND PROCESSING . . . . . . . . . . . 18

A. Particle Image Velocimetry . . . . . . . . . . . . . . . 18

B. PIV Post-Processing . . . . . . . . . . . . . . . 21

C. Measurement Uncertainties . . . . . . . . . . . . . 22

D. Conditional Averaging Methodology . . . . . . . . . . . . 22

E. Phase Averaging . . . . . . . . . . . . . . . . 24

IV RESULTS AND DISCUSSION . . . . . . . . . . . . . . 28 
A. Instantaneous Flow-Field Features . . . . . . . . . . . 28

B. Time-Averaged Properties . . . . . . . . . . . . 31

C. Statistical Analysis . . . . . . . . . . . . . . . . . 40

D. Conditionally Averaged Velocity Fields . . . . . . . . . . . 45

E. Phase-Averaged Representation . . . . . . . . . . . . . . . 45

F. Phase Averaging Morphology . . . . . . . . . . . . . . . . 49

V CONCLUSIONS \& RECOMMENDATIONS . . . . . . . . . . . 54

A. Summary of Results . . . . . . . . . . . . . . 54

B. Future Work . . . . . . . . . . . . . 55

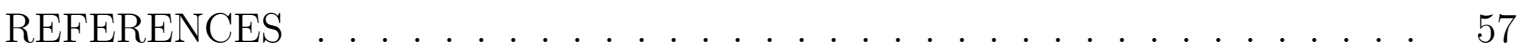




\section{LIST OF FIGURES}

FIGURE

1 Representative angles-of-attack on the blades of a rotor. The blade is rotating counterclockwise. . . . . . . . . . . . .

2 Sketch of $(a)$ light stall and $(b)$ deep stall. Figure taken from

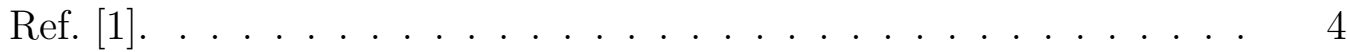

3 Qualitative description of the events of dynamic stall taken from Carr [2] . . . . . . . . . . . . . . . . . . . . .

$4 \quad$ Rendering of the experimental arrangement. Inset image is a graphical representation of the field-of-view at an angle of attack $\alpha=16.7^{\circ}$ with the origin of the coordinate system being the leading edge. Note the airfoil was painted with a black matte finish in order to minimize laser light reflections. . . . . . . . .

$5 \quad$ Present experimental conditions overlayed on a lift curve hysteresis loop taken from experiments conducted by Sheng et al [3-5]. The experimental conditions are similar to those used in the present study. The phases of dynamic stall are indicated which include: NDS - no dynamic stall effect, LDS - extension of linear lift curve slope, and NLDS - non-linear increase in lift curve slope. Note that the error bars are used as a guide to indicate the range the present data are projected to fall within. . . .

$6 \quad$ A graphical summary of some previous dynamic stall experiments reported in the literature. Figure adapted from Choudhouri \& Knight [6]. The present study is showed in bold. Note that several different types of airfoil are considered. $[5-15] \ldots \ldots$.

$7 \quad$ A comparison between the experimental and theoretical waveform with the error, $\Delta \alpha$, shown. Locations where statistical data were acquired are also indicated. The measured wing motion shows 1 in 8 data points for clarity. . . . . . . . . . . 
Instantaneous flow-field structure in the leading edge region of a generic angle of attack above static stall angle. (a) static stall $(b)$ upstroke instant with the flow being delayed above static stall angle $(c)$ downstroke image with separation of the flow-field evident . . . . . . . . . . . . . . . . .

9 Instantaneous flow-field structure in the leading edge region. $\alpha=16.7^{\circ}$ static with $(a)$ showing an instant with the flow fully attached, (b) complicated separated flow with few distinct features, and $(c)$ separated flow with dominant visible vortical structures. . . . . . . . . . . . . . . . . . . . .

10 Instantaneous flow-field structure in the leading edge region. $\alpha=22.4^{\circ}$ upstroke case with $(a)$ showing an instant with the flow fully attached, (b) complicated separated flow with few distinct features, and $(c)$ separated flow with dominant visible vortical structures. . . . . . . . . . . . . . . . . . . . . .

11 Time-averaged properties of the NACA 0012 airfoil at a static angle-of-attack $\alpha=16.7^{\circ}$. (a) Mean velocity $\bar{u} / U$, (b) Reynoldsaveraged shear-stress $\overline{u^{\prime} v^{\prime}} / U^{2},(c)$ Reynolds-averaged streamwise normal stress $\overline{u^{\prime} u^{\prime}} / U^{2},(d)$ Reynolds-averaged transverse normal stress $\overline{v^{\prime} v^{\prime}} / U^{2}$. . . . . . . . . . . . . . . . . . . . . .

12 Time-averaged properties of the NACA 0012 airfoil in the upstroke configuration at an angle-of-attack $\alpha=16.7^{\circ}$. (a) Mean velocity $\bar{u} / U,(b)$ Reynolds-averaged shear-stress $\overline{u^{\prime} v^{\prime}} / U^{2},(c)$ Reynolds-averaged streamwise normal stress $\overline{u^{\prime} u^{\prime}} / U^{2},(d)$ Reynoldsaveraged transverse normal stress $\overline{v^{\prime} v^{\prime}} / U^{2}$. . . . . . . . . . . . . . .

13 Time-averaged properties of the NACA 0012 airfoil at static angle-of-attack $\alpha=16.7^{\circ}$. (a) Mean velocity $\bar{u} / U$, (b) Reynoldsaveraged shear-stress $\overline{u^{\prime} v^{\prime}} / U^{2},(c)$ Reynolds-averaged streamwise normal stress $\overline{u^{\prime} u^{\prime}} / U^{2},(d)$ Reynolds-averaged transverse normal stress $\overline{v^{\prime} v^{\prime}} / U^{2} \ldots \ldots \ldots \ldots \ldots$ 
14 Time-averaged properties of the NACA 0012 airfoil at a static angle-of-attack $\alpha=22.4^{\circ}$. (a) Mean velocity $\bar{u} / U$, (b) Reynoldsaveraged shear-stress $\overline{u^{\prime} v^{\prime}} / U^{2},(c)$ Reynolds-averaged streamwise normal stress $\overline{u^{\prime} u^{\prime}} / U^{2},(d)$ Reynolds-averaged transverse normal stress $\overline{v^{\prime} v^{\prime}} / U^{2}$. . . . . . . . . . . . . . . . . 36

15 Time-averaged properties of the NACA 0012 airfoil in the upstroke configuration at an angle-of-attack $\alpha=22.4^{\circ}$. (a) Mean velocity $\bar{u} / U,(b)$ Reynolds-averaged shear-stress $\overline{u^{\prime} v^{\prime}} / U^{2},(c)$ Reynolds-averaged streamwise normal stress $\overline{u^{\prime} u^{\prime}} / U^{2},(d)$ Reynoldsaveraged transverse normal stress $\overline{v^{\prime} v^{\prime}} / U^{2}$. . . . . . . . . . . . . . .

16 Time-averaged properties of the NACA 0012 airfoil in the downstroke configuration at an angle-of-attack $\alpha=22.4^{\circ}$. (a) Mean velocity $\bar{u} / U,(b)$ Reynolds-averaged shear-stress $\overline{u^{\prime} v^{\prime}} / U^{2}$, (c) Reynolds-averaged streamwise normal stress $\overline{u^{\prime} u^{\prime}} / U^{2},(d)$ Reynolds-averaged transverse normal stress $\overline{v^{\prime} v^{\prime}} / U^{2}$. . . . . . . . .

17 Statistical analysis of the separated flow region. (left) Probability density distribution of reversed flow $P(u<0)$, (right) probability density function of separation location $x_{s e p} / c$. Results show (top row) $\alpha=16.7^{\circ}$ static, (middle row) $\alpha=16.7^{\circ}$ upstroke, (bottom row) $\alpha=16.7^{\circ}$ downstroke. . . . . . . . . . .

18 Statistical analysis of the separated flow region. (left) Probability density distribution of reversed flow $P(u<0)$, (right) probability density function of separation location $x_{s e p} / c$. Results show (top row) $\alpha=22.4^{\circ}$ static, (middle row) $\alpha=22.4^{\circ}$ upstroke, (bottom row) $\alpha=22.4^{\circ}$ downstroke. . . . . . . . . . . .

19 Conditional averaged perturbation fields for the $\alpha=16.7^{\circ}$ static (top row) and downstroke (bottom row) configuration. $(a, c)$ Conditional average of upstream separation, $(b, d)$ conditional average of downstream separation. Note both fields are mean subtracted. . . . . . . . . . . . . . . . . . 
20 Spectral and Hilbert transform analysis. The three rows represent different reference locations within the free-stream where the Hilbert transform was computed. (Left column) Power spectral density of the reference signal $v(t)$, with original signal inset. (Right column) Hilbert phasor diagram. Measurement locations: top row $(-0.027 x / c, 0.089 \mathrm{y} / \mathrm{c})$, middle row $(0.00$ $x / c, 0.069 \mathrm{y} / \mathrm{c})$, and bottom row $(0.022 x / c, 0.085 \mathrm{y} / \mathrm{c})$ referenced to $\alpha=16.7^{\circ}$ coordinate system. . . . . . . . . . . .

21 Selected phase-ensembled realizations showing dynamic stall flow-field evolution displayed with streamlines and velocity vectors at a skip of 1 in 8 in the streamwise direction and 1 in 2 in the transverse direction. Approximate angle-of-attack and phase angle are noted in the figure. Field-of-view is $0.16 c$ $\mathrm{x} 0.1 c$. The airfoil masks are representative and for illustration only. 


\section{LIST OF TABLES}

TABLE $\quad$ Page

1 Previous experimental and numerical campaigns . . . . . . . . . . 14

2 Estimate of experimental uncertainties. Definitions are provided in text . . . . . . . . . . . . . . . . . 23 


\section{CHAPTER I}

\section{INTRODUCTION}

\section{A. Dynamic Stall Overview}

Dynamic stall is an effect inherent to dynamical systems where a rapid change in the angle-of-attack of the airfoil can delay the stall angle. This unsteady aerodynamic

phenomenon was first found on helicopters and studied by Harris and Pruyn [16]. The study was developed due to an inability to accurately calculate the lift on high speed helicopters. It was found this problem was rectified if additional lift was present on the rotor in the retreating blade configuration. Furthermore, Ham and Garelick [17] found that additional lift was generated on a rapidly pitching airfoil due to the generation of a vortical structure. These works laid the foundation for decades of research that would be conducted in this area.

The unsteady phenomenon, coined dynamic stall, has attracted significant interest because of its potential to enhance the maneuverability and performance of aircraft, as well as its intimate connection with helicopter aerodynamics. Yet, despite intensive efforts, it has so far resisted a complete physical description due to a unique combination of flow unsteadiness, three-dimensionality, nonlinearity, and inviscid/viscous interaction $[2,18,19]$. An airfoil that experiences an unsteady increase in angle-of-incidence that carries it beyond its static stall angle is known to develop an increase in lift, without a noticeable change in lift-curve slope [11]. In particular, this behavior has been observed on helicopter rotor blades, where substantial changes in angle-of-attack occur in order to maintain stable flight due to varying velocity profiles on approaching and retreating blades as shown in Figure 1. 


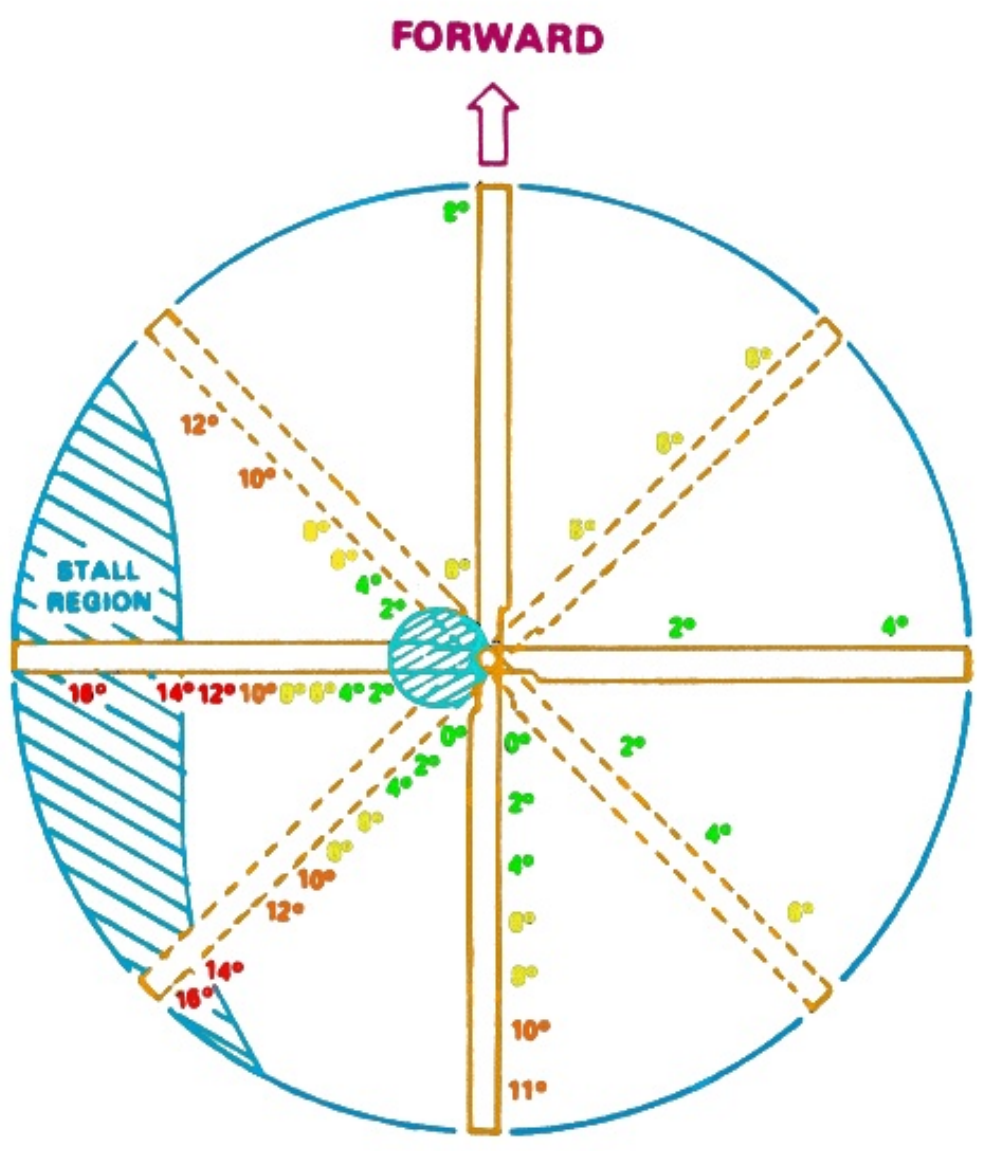

Fig. 1: Representative angles-of-attack on the blades of a rotor. The blade is rotating counterclockwise.

Fortunately, it is now known that the dynamic stall process on a helicopter blade may be experimentally reproduced on a pitching airfoil in a two-dimensional flow [20-22]. In this case, stall delay is an important phenomenon, because the overall performance of a rotor is limited by the stall risk on the retreating blades indicated in Figure 1.

Dynamic stall can be divided into two general stages, light and deep stall which are sketched in Figure 2. Light stall has many of the characteristics of static stall except that there is a growing hystersis in the airloads and the area of viscous inter- 
action is of the order of the airfoil thickness. Conversely, deep stall is characterized by significantly higher $C_{L}, C_{D}$, and $C_{M}$ compared to the static case, the presence of strong vortical structures, and the area of viscous interaction being of the order of the airfoil chord [1]. The deep stall regime can be further broken down as: gradual turbulent trailing edge stall, leading-edge stall caused by the abrupt breakdown of turbulent flow, or leading edge stall caused by the bursting of a laminar bubble. Ericsson and Reding [23] found the turbulent trailing edge stall to be characterized by reversed flow gradually moving forward from the trailing edge, while the laminar bubble stall was defined by an ejection of the boundary layer from the surface in a rapid motion from the bursting of a laminar bubble. The various subdivisions of dynamic stall are all characterized by a vortex shedding phenomenon and the progression of reversed flow from the trailing edge, but the leading edge label can be thought of as describing a rapid action rather than a gradual roll off [24].

The early phases of dynamic stall research focused significantly on experiments and semi-empirical modeling of the fluid dynamic forces and moments [25] in part due to the minimal computational resources available. This led to an intensive effort to identify the influence of several key parameters, such as Reynolds number, Mach number, reduced frequency, and mean pitch angle, on the dynamic stall process $[1$, $2,6,11]$. Further information on the background of dynamic stall research can be found in the review articles of McCroskey [1], Carr [2], Carr and McCroskey [26] and Ekaterinaris and Platzer [27].

\section{B. Conceptual Model}

The complexity of the dynamic-stall phenomenon has been outlined in detail by Carr [2], who presents a conceptual overview of the various stages of dynamic stall 
(a) LIGHT STALL

TRAILING-EDGE SEPARATION

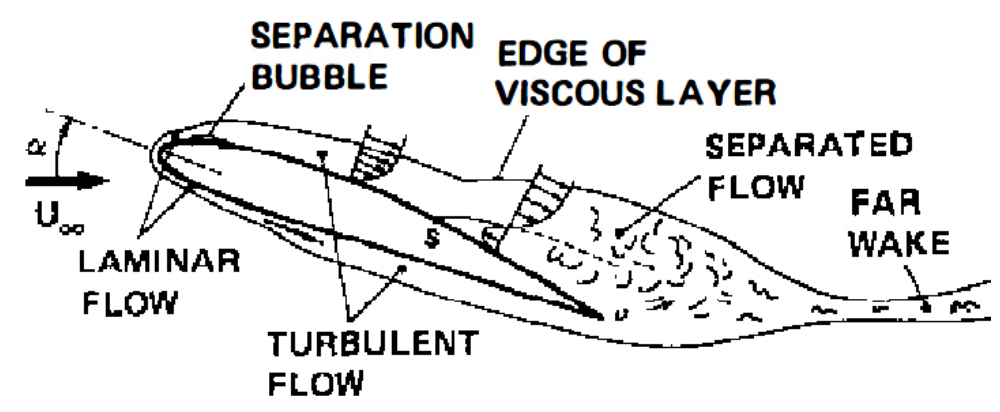

- STRONG INTERACTION

- VISCOUS LAYER = O(AIRFOIL THICKNESS)

(b) DEEP STALL

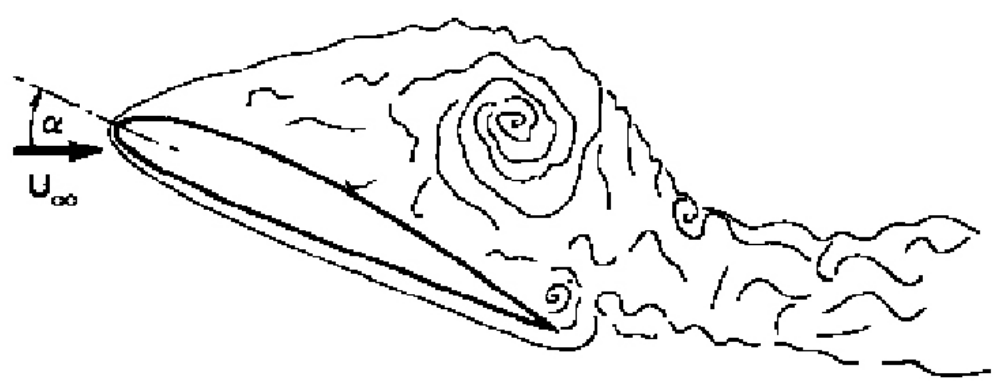

- VORTEX DOMINATED

- VISCOUS LAYER = O (AIRFOIL CHORD)

Fig. 2: Sketch of $(a)$ light stall and (b) deep stall. Figure taken from Ref. [1].

events shown in Figure 3. Although the specifics vary with airfoil shape, Reynolds number, and Mach number etc., the following description is useful for developing a qualitative understanding of the general dynamic stall events reported in the present paper.

Beginning from the flow being fully attached on the airfoil at low angle-of-attack, the first appearance of flow-reversal occurs typically near the trailing edge, when the static stall angle is exceeded. This is followed by large eddies that develop within the shear-layer of the airfoil. With further increase in angle of attack, flow reversal begins 


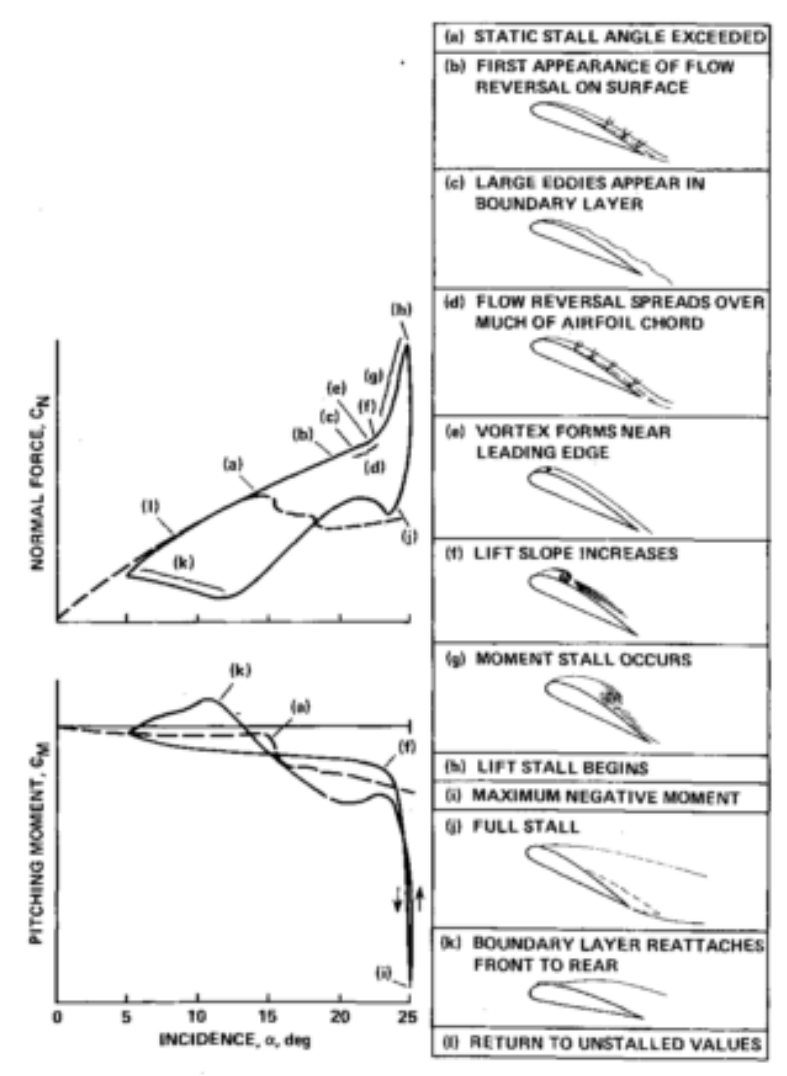

Fig. 3: Qualitative description of the events of dynamic stall taken from Carr [2].

to spread rapidly over much of the airfoil surface. At some point in the dynamics, a vortex forms near the leading edge. The precise conditions under which the formation of this vortex occurs still remains an open question. The leading edge vortex continues to grow at the leading edge until at a certain instant, it detaches from the surface of the airfoil and begins to convect downstream, staying near the surface of the airfoil. As the vortex moves over the surface, its size increases, leading to further increases in lift and negative pitching moment. With further increase in angle of attack, the vortex passes off the rear of the airfoil, leading to a peak in the negative pitching moment and a sudden loss of lift. The airfoil is now stalled and under an extensive amount of flow separation, characterized by reversed flow over the entire wing. As a result, 
this causes a large hysteresis loop in the lift, drag, and pitching moment curves with angle-of-attack. With a sufficient reduction in angle-of-attack, the boundary layer will again form on the airfoil, typically beginning to reattach from the leading edge. The literature suggests that when the leading edge vortex develops the wing enters a non-linear lift region, as indicated in Figure 5 [2]. This vortex grows in strength, detaches from the surface, and convects downstream.

\section{Motivation}

The Texas A\&M Dynamic Stall Facility was created to improve the understanding, predictability, and controllability of vortex-dominated unsteady flows as related to future Army weapon endeavors. The physics of these flows are Mach and Reynolds number dependent, and hence research in this area needs to be preformed at realistic flight conditions. Furthermore, detailed, experimental, non-intrusive measurements of velocity were also listed as needed in the separating region to yield new phenomenological understanding.

Drs. L. Carr and W. McCroskey at the request of Texas A\&M focused the requirements into more specific topics as they relate to dynamic stall control strategies. These topics were: (1) lack of understanding of the basic vortex dynamics with large pressure gradients, (2) uncertainties of applying quasi-steady turbulence models to dynamic stall problems, (3) influence of strong adverse pressure gradients on the turbulence models, and (4) the quantification and prediction of transition from laminar to turbulent flow. The current research focuses on the first point, but the data acquired can be used as a database for CFD comparisons relating to theories on the second and third point.

Historically, the dynamic stall model mentioned by Carr [2] has been developed 
using a variety of techniques including instantaneous pressure, interferometry, and normal force data. While instructive, direct quantitative visualization of the evolution of this vortex has been lacking [28], which may lead to details of the formation being lost. This process has also posed significant difficulties for computational fluid dynamics (CFD) modeling of the problem, since predictions involving realistic Reynolds numbers will only contribute toward improved flow-field understanding if progress is made in the ability to numerically predict boundary layer transition to turbulent flow $[8,27,29]$. Current CFD simulations appear to have the ability to accurately predict lift, drag, or the moment loop, but generally fail to reproduce two or all three components simultaneously [27].

\section{Research Objectives and Scientific Contribution}

A strong motivation therefore exists to experimentally investigate the leading edge flow structure of a dynamically pitching airfoil, both instantaneously and statistically, using whole-field quantitative techniques. The data acquired will improve the physical understanding of the leading edge vortex structure which implies an extended model suggested in section E. Additionally the turbulence statistics acquired will form a database for CFD model validation which are being increasingly used in dynamic stall applications. The geometry used for this experimental work was the NACA 0012 airfoil due to its canonical nature and to maximize the reach of the experimental database for CFD comparison.

The data acquired and presented in this thesis is as follows: Phase locked static, upstroke motion, and downstroke motion at an $\alpha=16.7^{\circ}$ and $\alpha=22.4^{\circ}$. Additionally

phase averaged results are presented from the entire cycle of motion. The method for acquisition and analysis are discussed in chapter III section E. 


\section{E. Previous Work in Texas A\&M DSF}

Mean and turbulence properties have been obtained by Sahoo et al. [30] under similar flow conditions to the present study. However, due to experimental errors, the previous work drew incorrect conclusions. When these errors were remedied by the present authors, those results exhibited qualitatively similar trends to the ones reported in this study. Therefore, this work is considered to be the new metric for comparison to the NACA 0012 geometry.

Vanelli conducted similar experiments on a Sikorsky SSC-A09 airfoil. Mean and turbulence properties were computed for this geometry. Further information on this campaign can be found in reference [31].

\section{F. Thesis Outline}

Chapter II contains detailed information on the facility that the testing was conducted in and the experimental conditions that were tested. Chapter III contains information on the diagnostics that were used and the data analysis techniques. Chapter IV contains the results from the selected angles-of-attack and the discussion that was derived from the testing. Chapter V contains concluding remarks along with recommendations for future testing that would enhance the work that was recently completed. 


\section{CHAPTER II}

\section{EXPERIMENTAL SETUP}

\section{A. Facility}

Experiments were conducted in the dynamic stall facility (DSF), located in the Oran W. Nicks low-speed wind-tunnel at Texas A\&M University. The standard testsection for this facility is $2.13 \mathrm{~m} \times 3.05 \mathrm{~m}$, but a specially designed insert was constructed so that the dimensions for this experiment were $2.13 \mathrm{~m} \times 2.13 \mathrm{~m}$. The Mach number range in the test section is $0.1-0.3$. In addition there is a secondary insert that results in a test section size of $1.52 \mathrm{~m} \times 2.13 \mathrm{~m}$. This insert was designed to extend the Mach number range to 0.4 in order to study compressibility effects. More information about the design and calibration of the dynamic stall facility can be found in reference [32]. Recent improvements to the infrastructure have a possibility of increasing Mach number range up to 0.5 or greater. Later work will quantify how the new improvements effect operational ranges.

A NACA 0012 airfoil spanned the full width of the test-section, which provided an aspect ratio of approximately 5. It was demonstrated via an array of tufts placed on the airfoil surface that the flow under the present experimental conditions was symmetric at the angles-of-attack considered. The airfoil section was constructed from Al 5052, and had a stainless steel shaft running through the quarter chord. The shaft was $0.0831 \mathrm{~m}$ in diameter, and was supported with a bearing ring on one end, and two on the other, which were representative of simply and fixed supports, respectively. This set-up minimized the wing deflections, which were less than $2 \mathrm{~mm}$ $(<0.1 \%$ of the span $)$ 
A Parker Fluids Power Systems brand hydraulic pump system with a $22.4 \mathrm{~kW}$ motor was used to control the dynamic pitching of the airfoil at a desired frequency. The hydraulic actuator was mounted to one end of the stainless steel shaft via a moment arm, and was controlled by a hydraulic valve in combination with a proportionalintegral-derivative (PID) controller. The PID controller was used to produce the desired angle-of-attack wave-form, which for the present study was a sinusoid. This controller was programmed using RMCWin software, with careful selection of the proportional, integral, and differential gains in order to maintain accurate control over the wing.

The static angle-of-attack could be measured using an inclinometer to $\pm 0.05^{\circ}$. The angle-of-attack of the pitching airfoil was measured using a linear voltage displacement sensor, which was mounted within the hydraulic cylinder. The uncertainty in position, $\Delta \alpha$, was determined by taking the maximum deviation between the theoretical command signal and actual wing position, which was found to be $\pm 0.2^{\circ}$. Thus, a maximum uncertainty of $\pm 0.25^{\circ}$ is quoted in the present study for determining the angle-of-attack. For the upstroke and downstroke cases, the PIV images were phase-locked at static angles-of-attack, $\alpha=16.7^{\circ}$ and $22.4^{\circ}$.

During testing, it was found that there is system lag in the hardware that controls the phase locking of the wing, which if left uncorrected led to a difference between the static, upstroke, and downstroke images. The lag in the system for one angle-of-attack was found to be $5 \mathrm{~ms}$ between the upstroke and downstroke which equates to $0.5^{\circ}$. It is postulated that the lag is both a function of the wing pitching frequency and angle-ofattack due to the acceleratory and decelatory corrections made by the PID controller. Before final data was acquired, the triggers for the upstroke and downstroke were adjusted such that they matched the static images, and were therefore at the noted angle of attack. 


\section{B. Experimental Conditions}

The aim of the present work is to experimentally investigate the leading edge flow structure of a dynamically pitching NACA 0012 at conditions representative of a realistic rotor in flight in the deep stall regime. Limitations in the experimental facility led to a compromise of the parameters of interest. Specifically, maintaining a reduced frequency of $k(=\pi f c / U)=0.1$, where $f$ is the pitching frequency, $f=2.5 \mathrm{~Hz}$, and $U$ is the free-stream velocity, $U=35 \mathrm{~m} \mathrm{~s}^{-1}$, was important in the design of the experiment. Compressibility effects were not the focus of this paper, therefore a low Mach number $(\mathrm{M}=0.1)$ was chosen which rendered the flow effectively incompressible. The anglesof-attack being studied are, $\alpha=16.7^{\circ}, 22.4^{\circ} \pm 0.25^{\circ}$, at a Reynolds number, $R e_{c}=$ $1 \times 10^{6}$ (where $c$ is the airfoil chord, $c=0.45 \mathrm{~m}$ ). A conception of the experimental arangement can be seen in figure 4 with the field-of-view indicated in the insert.

The angle-of-attack $\alpha=16.7^{\circ}$ and $22.4^{\circ}$ were chosen to illustrate the different dynamic stall regions as depicted in Figure 5, where the normal force data is taken from Ref. 11 and 12 at $R e_{c}=1.5 \times 10^{6}$ and $M=0.12$. These conditions are very similar to the present study, except for the difference in mean pitch angle (cf. $\alpha_{\circ}=15^{\circ}$ to $\alpha_{\circ}=21^{\circ}$ in the present study). By matching all parameters except for the mean pitch angle, $\alpha_{\circ}$, the flow structure is expected to be the same until motion of the wing changes [11]. Using this idea, on the upstroke a comparison in the data can be made until $\approx 24^{\circ}$. The data in the cycle past this point and all of the downstroke cannot be compared due to differences in maximum angle of attack. Once the flow reattaches, the comparison is valid again and therefore the lower bound is conservatively estimated to be $\approx 13^{\circ}$ on the upstroke. Therefore a range from $13^{\circ}-24^{\circ}$ is expected to maintain similar lift characteristics which brackets the data acquired.

From figure 5 it can be seen that the $16.7^{\circ}$ case is well within the LDS regime, 


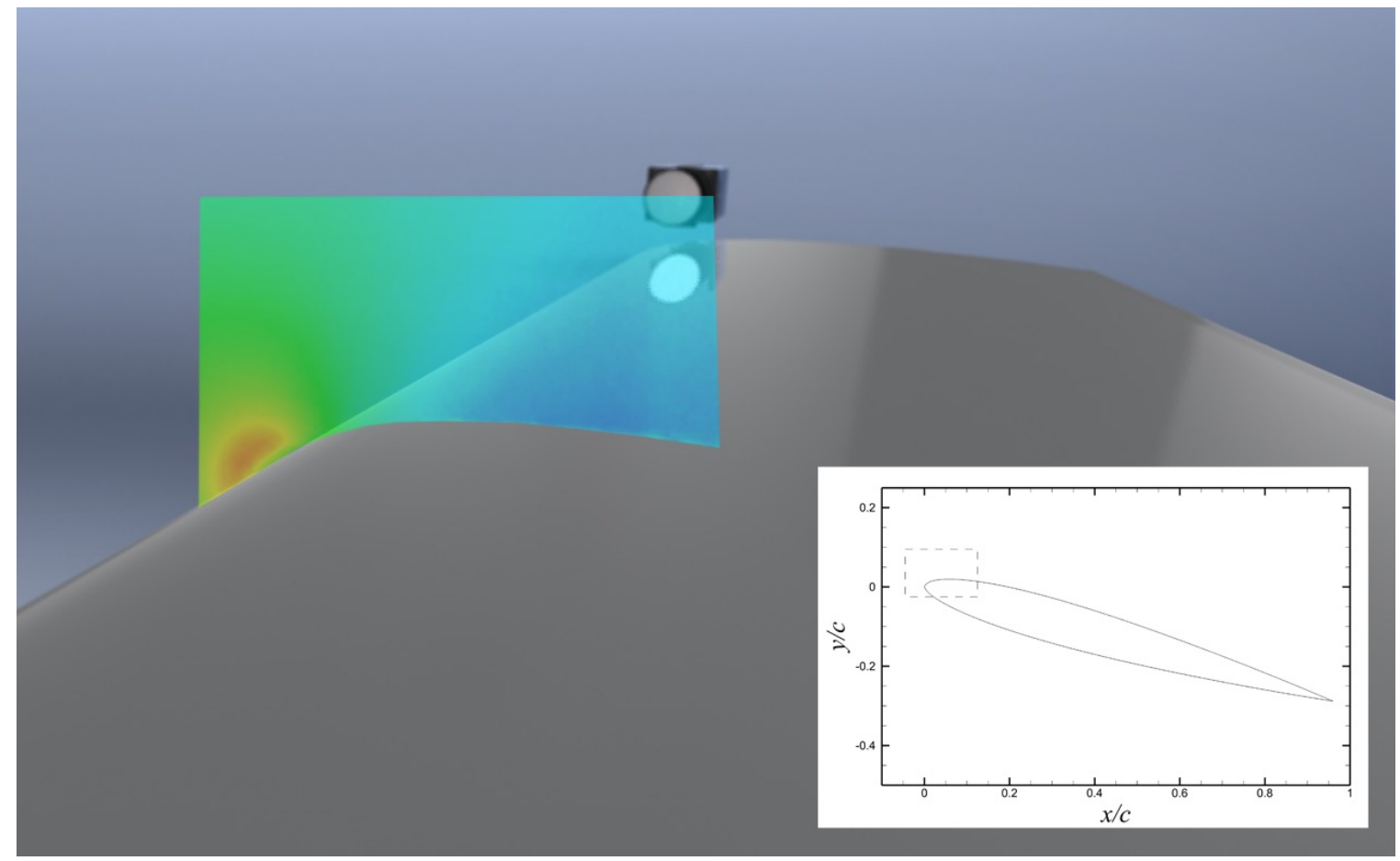

Fig. 4: Rendering of the experimental arrangement. Inset image is a graphical representation of the field-of-view at an angle of attack $\alpha=16.7^{\circ}$ with the origin of the coordinate system being the leading edge. Note the airfoil was painted with a black matte finish in order to minimize laser light reflections. 


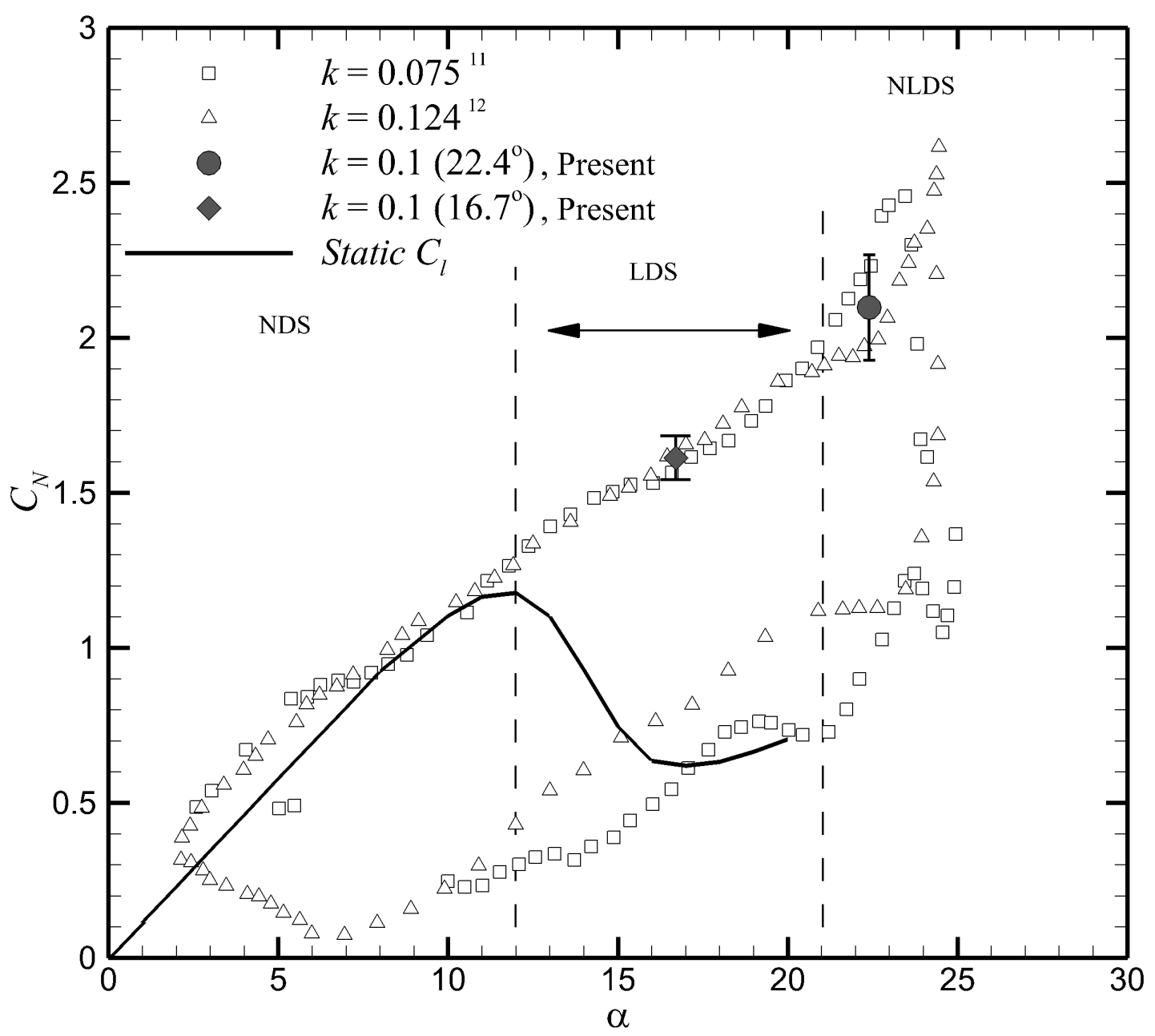

Fig. 5: Present experimental conditions overlayed on a lift curve hysteresis loop taken from experiments conducted by Sheng et al [3-5]. The experimental conditions are similar to those used in the present study. The phases of dynamic stall are indicated which include: NDS - no dynamic stall effect, LDS - extension of linear lift curve slope, and NLDS - non-linear increase in lift curve slope. Note that the error bars are used as a guide to indicate the range the present data are projected to fall within. 
characterized by an extension of the linear lift region well beyond the static stall angle. In contrast, the $22.4^{\circ}$ case was chosen to fall within the non-linear dynamic stall (NLDS) region. Conventionally this region corresponds to the formation of a leading edge vortex, which is producing the nonlinear effect evident at this angle-ofattack.

Table 1: Previous experimental and numerical campaigns

\begin{tabular}{|c|c|c|c|c|}
\hline Author & Mach & $k$ & Reynolds & Technique \\
\hline Ahmed et al. [7] & 0.3 & 0.05 & $5.4 \times 10^{5}$ & Schlerien, LDV, PDI \\
\hline Choudhouri et al. [8] & 0.2 & 0.2 & $1.00 \times 10^{4}$ & Computational \\
\hline Crisler et al. [9] & 0.23 & 0.03 & $2.80 \times 10^{5}$ & P-PIV \\
\hline Crisler et al. [9] & 0.45 & 0.03 & $6.20 \times 10^{5}$ & P-PIV \\
\hline Ekaterinaris et al. [10] & 0.45 & 0.05 & $1.5 \times 10^{6}$ & PDI \& Schlerien \\
\hline Choudhouri et al. [6] & 0.5 & 0.1 & $1.00 \times 10^{4}$ & Computational \\
\hline Choudhouri et al. [6] & 0.2 & 0.1 & $1.00 \times 10^{4}$ & Computational \\
\hline McAlister et al. [11] & 0.09 & 0.25 & $2.50 \times 10^{6}$ & Hotwire \& Surface Pressure \\
\hline McAlister et al. [11] & 0.09 & 0.15 & $2.50 \times 10^{6}$ & Hotwire \& Surface Pressure \\
\hline McCroskey et al. [12] & 0.3 & 0.2 & $4.00 \times 10^{6}$ & Hotwire \& Surface Pressure \\
\hline Mulleners et al. [13] & 0.14 & 0.1 & $9.20 \times 10^{5}$ & TR-PIV \& Surface Pressure \\
\hline Sheng et al. [3] & 0.12 & 0.075 & $1.5 \times 10^{6}$ & Normal Force \\
\hline Sheng et al. [5] & 0.12 & 0.124 & $1.5 \times 10^{6}$ & Normal Force \\
\hline Wernert et al. [15] & 0.1 & 0.15 & $3.73 \times 10^{5}$ & P-PIV \& Visualizations \\
\hline Pruski & 0.1 & 0.1 & $1.00 \times 10^{6}$ & P-PIV High Resolution \\
\hline
\end{tabular}




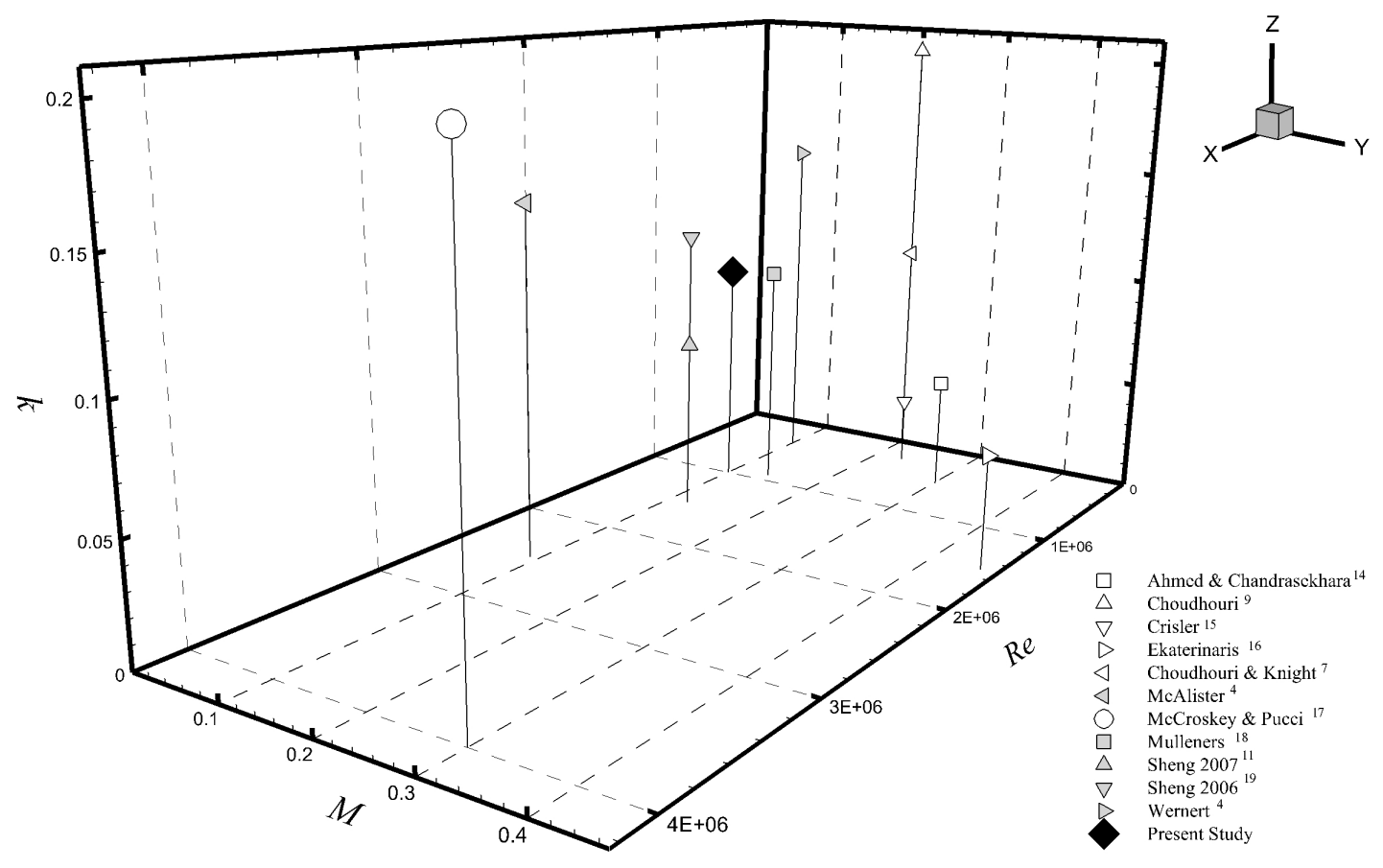

Fig. 6: A graphical summary of some previous dynamic stall experiments reported in the literature. Figure adapted from Choudhouri \& Knight [6]. The present study is showed in bold. Note that several different types of airfoil are considered. [5-15] 
The present experimental conditions are indicated in Figure 6 and Table 1, along with a selection of other relevant experiments reported in the literature. It is evident that while realistic conditions are not quite matched, the present experimental conditions are not unusual.

Figure 7 illustrates a comparison between the waveform obtained from the PID controller, and the theoretical waveform, described by $\alpha=21-10 \sin \omega t$, where $\omega=$ $2 \pi f$, and the oscillating frequency $f$, was chosen to be $f=2.5 \mathrm{~Hz}$ in order to give a reduced frequency of approximately $k=0.1$. The maximum discrepancy between the experimental and theoretical $\alpha$ occurs at the top of the cycle. The error, $\Delta \alpha$, is also indicated and is repeatable from cycle-to-cycle. 


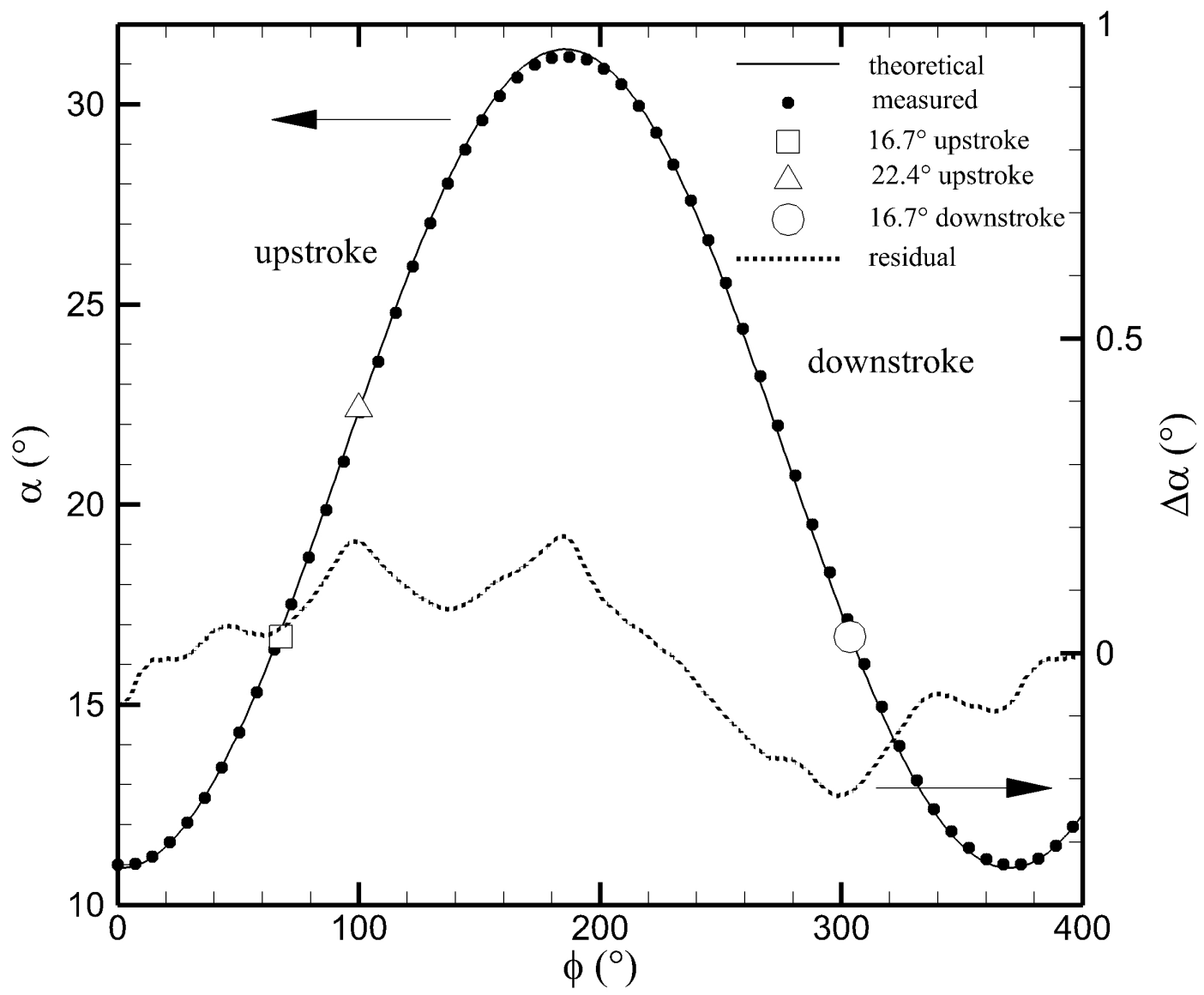

Fig. 7: A comparison between the experimental and theoretical waveform with the error, $\Delta \alpha$, shown. Locations where statistical data were acquired are also indicated. The measured wing motion shows 1 in 8 data points for clarity. 


\section{CHAPTER III}

\section{DIAGNOSTICS AND PROCESSING}

\section{A. Particle Image Velocimetry}

Particle Image Velocimetry (PIV) enables the instantaneous visualization of the flow-field, while providing measurement of the instantaneous velocity/vorticity distribution. Two-component PIV was employed in the present study, in order to provide whole-field velocimetry data of the leading edge region. The flow was seeded with MDG neutral fluid, a mineral oil mixture, which was aerosolized using a MDG Max 5000 fog generator. Given the closed-circuit nature of the facility, fine adjustment of the seeding density was possible, and very little shot-to-shot variation in the seeding density of the images was observed.

According to the manufacturer, the nominal particle diameter ranges between $d_{p}=0.5-0.7 \mu \mathrm{m}$ and the particle density is $\rho_{p}=850 \mathrm{~kg} \mathrm{~m}^{-3}$. Thus, following Menon and Lai [33], the $3 \mathrm{~dB}$ temporal response of the particles was estimated to be $\tau_{p} \approx 1.2$ $\mu \mathrm{s}$, or a frequency response of $f_{p} \approx 130 \mathrm{kHz}$. The Stokes number, St, may be used to quantify the particle-tracking performance. The Stokes number is defined by $S t$ $=\tau_{p} / \tau_{f}$, where $\tau_{f}$ is the flow time-scale. In the present study, $\tau_{f}$ was taken to be $d / U$, where $d$ is the size of a characteristic eddy structure visualized in the shearlayer. The eddy structure was chosen because it was representative of the structure size being studied for formation of the dynamic stall vortex. The length scale of an eddy structure was chosen because it was difficult to unambiguously infer an incoming boundary layer thickness, and the boundary layer was not the chief spatial scale of interest. For the interested reader, the $16.7^{\circ}$ upstroke case yields a boundary layer 
height of approximately $2 \mathrm{~mm}$ after evolving $0.12 c$. Using a characteristic eddy size of approximately $9 \mathrm{~mm}$ (cf. Figure 6), results in a flow time-scale of $\tau_{f}=9 \times 10^{-3} / 35=$ $2.6 \times 10^{-4} \mathrm{~s}$, which gives a Stokes number of $S t \approx 0.005$. The particles are therefore considered to faithfully track the characteristic eddy sizes of interest. Using the criterion that $\mathrm{St}<0.1$, an experimental limit mentioned by Samimy and Lele [34], a structure with length scale $0.5 \mathrm{~mm}$ could be tracked, but should be considered an optimistic estimate.

The particles were illuminated using a New Wave Solo 120 XT dual-head Nd:YAG laser. This particular variant has $120 \mathrm{~mJ}$ of energy per pulse (4 ns pulse duration) at a wavelength of $532 \mathrm{~nm}$. The time separation between pulses was varied between 18 and $25 \mu \mathrm{s}$ in order to maintain a suitable pixel displacement of approximately 30 pixels. The acquisition rate of the particle image pairs was $15 \mathrm{~Hz}$ for the static experiments, $2.5 \mathrm{~Hz}$ for the dynamic experiments, and $13.75 \mathrm{~Hz}$ for the phase-averaged results discussed in chapter IV section F. The laser beam entered through the ceiling of the wind-tunnel using laser light optics. A knife-edge filter was used in order to remove the low-energy fringes and to produce a near top-hat light intensity distribution. Using a combination of spherical and cylindrical lenses, a thin laser sheet of approximately $0.5 \mathrm{~mm}$ thickness was formed at the airfoil surface. Laser light reflections from the airfoil were minimized by mounting the camera at a slight angle of attack relative to the laser sheet $\left(<2^{\circ}\right)$.

A rendering of the experimental setup is shown in Figure 4. The wind-tunnel walls have been removed for clarity. The camera was set up outside the wind-tunnel and particle images are acquired through a three inch optical flat. Originally a 11.4 $\mathrm{cm} \times 2.54 \mathrm{~cm}$ piece of Plexiglas was inserted into the leading edge of the airfoil which matched the profile of the NACA 0012. This was an attempt to further minimize laser light reflections. During testing it was found that reflections were actually increased, 
possibly due to refraction where the Plexiglas met with the aluminum. Therefore the insert was taped over with a black masking tape which substantially reduced the reflections. The entire wing was also painted matte black in order to minimize laser light reflections.

An additional concern during acquisition were the broadband vibrations transmitted through the facility being generated by the motion of the airfoil. These vibrations necessitated the isolation of the laser and camera equipment from the wind-tunnel structures through the use of additional supports and vibration damping materials. Though no data was acquired at the higher frequencies of motion, it was found that the vibrations increased substantially with the flapping frequency, but did not seem to change significantly with an increase in Mach number. It is worth noting that to maintain the reduced frequency at $k=0.1$, an increase in Mach number would require an increase in flapping frequency. The additional vibrations crated will have to be addressed in any future experiments to acquire results of similar fidelity.

The flow was imaged using a 14-bit Peltier-cooled Cooke PCO 1600 chargecoupled display $(\mathrm{CCD})$ camera, with a $1600 \times 1200$ pixel-size sensor. During the experiment, the camera was fixed to the laboratory reference frame. The camera was equipped with a Nikon f/4 70-300 mm lens. In the present study, a focal length of 266 $\mathrm{mm}$ was used in combination with an $\mathrm{f}$-stop of $\mathrm{f} \#=8$. In order to resolve the leading edge structures, the field-of-view was $75 \mathrm{~mm} \times 56 \mathrm{~mm}(0.17 c \times 0.12 c)$, resulting in a digital resolution of 21 pixels $\mathrm{mm}^{-1}$. The field-of-view is schematically depicted in Figure 4. This small field-of-view was necessary to resolve the subtle features involved in vortex formation. Additionally, future experiments should use a wider field-of-view to determine the state of the shear-layer. This will give a clear answer to the question of whether the airfoil is experience large scale separation or if there is a reattachment of the shear-layer outside the smaller field-of-view. In the absence of pressure data, 
the behavior of the shear-layer can indicate the state of stall on the surface of the airfoil.

The origin of the coordinate system used is located at the leading edge of the airfoil, with the x-axis taken to be along the wind-tunnel longitudinal axis (i.e., the undisturbed free-stream flow direction), and the y-axis normal to the wind-tunnel longitudinal axis. This seemed appropriate due to the type of analysis being used on the data. The disadvantage is that every angle of attack has a unique coordinate system in a global sense. This could have been rectified by using the quarter chord as the coordinate system, but that would have complicated the description of physical phenomenon due to the small field-of-view being employed in this study.

\section{B. PIV Post-Processing}

As mentioned earlier, the PIV system was phase-locked with the airfoil motion, and a total number $\mathrm{N} \approx 700$ image pairs were acquired for each test case, which was sufficient to create statistically meaningful results in post processing. Any attempt to get calculate higher-order statistics will require significantly more images. Particle images were processed using the LaVisions DaVis software 8.0. A four-step adaptive cross-correlation in sizes: $128 \times 128,64 \times 64,32 \times 32$, down to a final interrogation window size $16 \times 16$ pixels was used, in combination with an overlap factor of $50 \%$. The stepping procedure from the largest window size to the smallest window size was used in order to increase the validity of the vectors in the final window size, as well as to eliminate erroneous vectors at each pass during the correlation process. This results in a final vector spacing of approximately $0.37 \mathrm{~mm}(0.08 \% c)$. The first row of vectors near the surface occasionally gave unreliable results, therefore the second row, $0.75 \mathrm{~mm}$ from the surface, is considered the first row of reliable data (i.e. $0.37 \delta$ 
from the wall as an optimistic estimate).

LaVision's software package gives several options for gaussian weighting functions which can be used to porcess the data which inclue square, ellipse, circle, and adaptive. A case study was carried out on the data to determine which weighting function would give the smallest number of artifacts which seemed non-physical to the studied flowfield. The results of the study indicated the circular Gaussian weighting function as the best option becasue it minimized the effects of the edges of the interrogation windows overlapping with the profile of the airfoil. Vector validation was carried out using a standard median filter. The number of spurious vectors in the dataset was less than 5\%. These were removed and replaced using linear interpolation.

\section{Measurement Uncertainties}

A summary of the statistical uncertainties associated with a finite number of samples is provided in Table 2. The time-averaged and turbulence statistical uncertainties were calculated following the work of Benedict \& Gould [35]. The uncertainty in the probability density function of reversed flow, $P$, is approximated by the uncertainty in the mean flow $U$. The uncertainty in the conditionally averaged perturbation field increases relative to the normal stresses due to the reduced sample size.

\section{Conditional Averaging Methodology}

In order to isolate the subtle physical features of vortex formation, large scale motions that dominate the flow-field must be removed. In the case of unsteady flow separation, the dominant motion is the movement of the separation point along the surface of the airfoil. Therefore to see if any features are being obscured by the large scale motions, conditional averaging is implemented. 
Table 2: Estimate of experimental uncertainties. Definitions are provided in text

\begin{tabular}{cc}
\hline \hline Variable & Uncertainty \\
\hline $\bar{u} / U$ & $1.5 \%$ \\
$\overline{u^{\prime} u^{\prime}} / U^{2}, \overline{v^{\prime} v^{\prime}} / U^{2}$ & $5 \%$ \\
$\overline{u^{\prime} v^{\prime}} / u^{\prime} v_{r m s}^{\prime}$ & $15 \%$ \\
$P$ & $1.5 \%$ \\
$\tilde{\tilde{u}}_{1} / U, \tilde{\tilde{u}}_{2} / U$ & $7 \%$ \\
\hline \hline
\end{tabular}

The procedure is as follows: the separation location is determined in each image (if it occurs at all). The images are then sorted by separation location, from leading edge to trailing edge, such that images of similar flow-field separation characteristics are binned together. To avoid smoothing out small perturbations in the flow-field, the histograms, figure $17(\mathrm{~b}, \mathrm{f})$, were used to eliminate spurious data in the tails of the distribution. Any histogram bins containing less than $3 \%$ of the complete data set were removed, and the remaining data were analyzed by creating a conditional mean from the subset of images. The mean flow was then removed from the conditional averages leaving perturbation fields $\tilde{\tilde{u}}_{1}$ and $\tilde{\tilde{u}}_{2}$, representing the fluctuating velocity fields of when the separation point was significantly upstream and downstream, respectively. This procedure is summarized mathematically as:

$$
\begin{gathered}
\bar{u}(x, y)=\frac{1}{N} \sum_{i=1}^{N} u_{i}(x, y, t), \\
\tilde{\tilde{u}}_{1}=\frac{1}{n} \sum_{i=1}^{n} u_{i}(x, y, t)-\bar{u}(x, y),
\end{gathered}
$$




$$
\tilde{\tilde{u}}_{2}=\frac{1}{n} \sum_{i=N-n}^{N} u_{i}(x, y, t)-\bar{u}(x, y) .
$$

where $N=690$ and $n$ is the conditional average subset of fifty images. The latter was chosen as a balance between having a converged flow-field and having the points sufficiently close together to avoid obscuring subtle features.

\section{E. Phase Averaging}

Most of the results acquired were done at a specific angle of attack. While this is instructive for turbulence statistics, it lacks the information for how the flow-field behaves through the entire cycle of motion. Acquiring data throughout the entire motion of the wing can give insight into how the flow-field evolves into a particular angle of attack which may be of interest. Furthermore, any attempt to control the phenomenon of dynamic stall will require an understanding of how the flow-field reacts throughout the entire phase. The current capabilities of the Dynamic Stall Facility at Texas A\&M University do not allow for the acquisition of time resolved data which would be ideal for this type of study. Therefore in an attempt to maximize the resources available, a phase averaging procedure was implemented.

The dynamic stall process consists of a large-scale quasiperiodic component of boundary layer separation and reattachment, accompanied by a smaller-scale residual component, which can be conveniently characterized using a phase-averaging procedure. To accomplish this, velocity vector fields were acquired at $13.75 \mathrm{~Hz}$, which is a noninteger multiple of the $2.5 \mathrm{~Hz}$ harmonic flapping motion to ensure that the phase space representation was appropriately populated. Thus, for each complete airfoil oscillation cycle, approximately 5 snapshots were acquired. This sample rate allowed for resolution of frequencies present within the flow-field up to $6.88 \mathrm{~Hz}(\approx 2.5 f)$. The 
phase of each snapshot was determined using the triple signal decomposition approach of Hussain and Reynolds [36] in combination with the Hilbert transform, since the angle of attack was not tracked for each image pair [37]. Briefly, the instantaneous velocity (in this case the normal component $v$ for reasons which will be explained shortly) is decomposed via the standard two-component Reynolds decomposition:

$$
v(x, y, t)=\bar{v}(x, y)+v^{\prime}(x, y, t)
$$

where $\bar{v}$ is the mean flow component, and $v^{\prime}$ is the fluctuating component. For flows that are characterized by a periodic component, the fluctuating component can be further decomposed as:

$$
v(x, y, t)=\bar{v}(x, y)+\tilde{v}(x, y, t)+\hat{v}(x, y, t)
$$

where $\bar{v}$ is the mean flow component, $\tilde{v}$ is the periodic component, and $\hat{v}$ is the turbulent contribution to the fluctuation. The coherent portions are then combined such that there is a mean component and a fluctuating component, which gives

$$
v(x, y, t)=\langle v(x, y, \phi(t))\rangle+\hat{v}(x, y, t)
$$

where $v(x, \phi(t))$ is the phase-averaged velocity at a point in space and phase $\phi(t)$. The phase averaged velocity field is defined by

$$
\langle v(x, y, \phi(t))\rangle=\lim _{N \rightarrow \infty} \frac{1}{N} \sum_{n=1}^{N} v(x, y, \phi(t+m \tau)),
$$

where $\tau$ is the period of the wing motion. This representation is akin to Reynolds averaging except that instead of making a time-average on the entire data set, the data set is broken down into several segments with averages being computed in each 
segment. The Hilbert transformation was used to determine the phase of each instantaneous image. Although several approaches have been used in the literature, the Hilbert transform offers a robust method for unambiguously determining the phase variable. An additional advantage of this method is that an analytic signal is directly computed from the measured one, providing a direct method of determining the phase and amplitude. The Hilbert transformation of the fluctuating velocity is defined as

$$
H\left\{v^{\prime}(t)\right\}=\frac{1}{\pi} P \int_{-\infty}^{\infty} \frac{v^{\prime}(t)}{t-\tau} d \tau,
$$

where $P$ (not to be confused with the probability density function described earlier) is the Cauchy principal value defined by

$$
P=\int_{-\infty}^{\infty} f(x) d x \equiv \lim _{R \rightarrow \infty} \int_{-R}^{R} f(x) d x .
$$

This is required in the transformation because of the possibility of a singularity at time $t=\tau$. The Hilbert transform, $H\left\{v^{\prime}(t)\right\}$, and the velocity signal, $v^{\prime}(t)$, form a complex conjugate pair which define the analytic signal $Z(t)$ as

$$
Z(t)=v^{\prime}(t)+i H\left\{v^{\prime}(t)\right\}
$$

where $i$ is the imaginary number $\left(i^{2}=-1\right)$. In order to bring the phase variable into the analysis, Eq. 3.10 is rewritten in exponential form as

$$
Z(t)=a(t) e^{i \phi(t)},
$$

where $a(t)$ is the amplitude of the signal and $\phi(t)$ is the phase of the analytic function which are defined respectively as 


$$
\begin{gathered}
a(t)=\sqrt{v^{\prime}(t)^{2}+H\left\{v^{\prime}(t)\right\}^{2}}, \\
\phi(t)=\tan ^{-1} \frac{H\left\{v^{\prime}(t)\right\}}{v^{\prime}(t)} .
\end{gathered}
$$

The phase variable was derived from the fluctuating vertical component, $v(t)$, which was an arbitrary choice. The vertical component is sensitive to the wing motion and and enables the phase plot to be centered around zero instead of some mean value. The phase component gives a measure of the period of the cycle. That is, the difference between any two phase angles (i.e., equivalently, any two measurement times) is $\Delta \phi$. Thus, the number of $\Delta \phi$ 's within a circle (loosely) defines the period. 


\section{CHAPTER IV}

\section{RESULTS AND DISCUSSION}

\section{A. Instantaneous Flow-Field Features}

To provide an overview of the main phenomenological features observed, figure $8(a-c)$ depicts a series of instantaneous flow-fields for the static, upstroke, and downstroke cases, respectively. These instants have been chosen to illustrate the distinct instantaneous structure of the flow-field throughout the dynamic stall cycle. An airfoil above its static stall angle as shown in figure $8(a)$ displays a large region of reversed-flow over the surface of the airfoil, which is bounded by both the airfoil surface and the separated shear-layer. Within the shear-layer, discrete vortical structures are apparent with a size comparable to the shear-layer thickness, and with a vorticity component that is parallel to the spanwise direction. Animation of several instantaneous snapshots suggests that these vortical structures are shed from just downstream of the separation point. The vortices all appear to have the same clockwise (i.e., negative out-of-plane vorticity) rotational direction, and from the surrounding vectors, appear to entrain fluid from the external flow. In-between these vortical structures, saddle points (stagnation points) may also be observed, which are attributed to the co-rotating cancelling out of fluid motion between pairs of structures. The upstroke cycle shown in figure $8(b)$ is characterized by a fully attached flow-field [2]. Instantaneous snapshots of fully attached flow will occur on the upstroke until the leading edge vortex forms, causing a separation of the shear-layer from the surface which will be discussed more in section F. In contrast, the downstroke configuration in figure

$8(c)$ displays a large region of separated flow, typically exhibiting the characteristics 

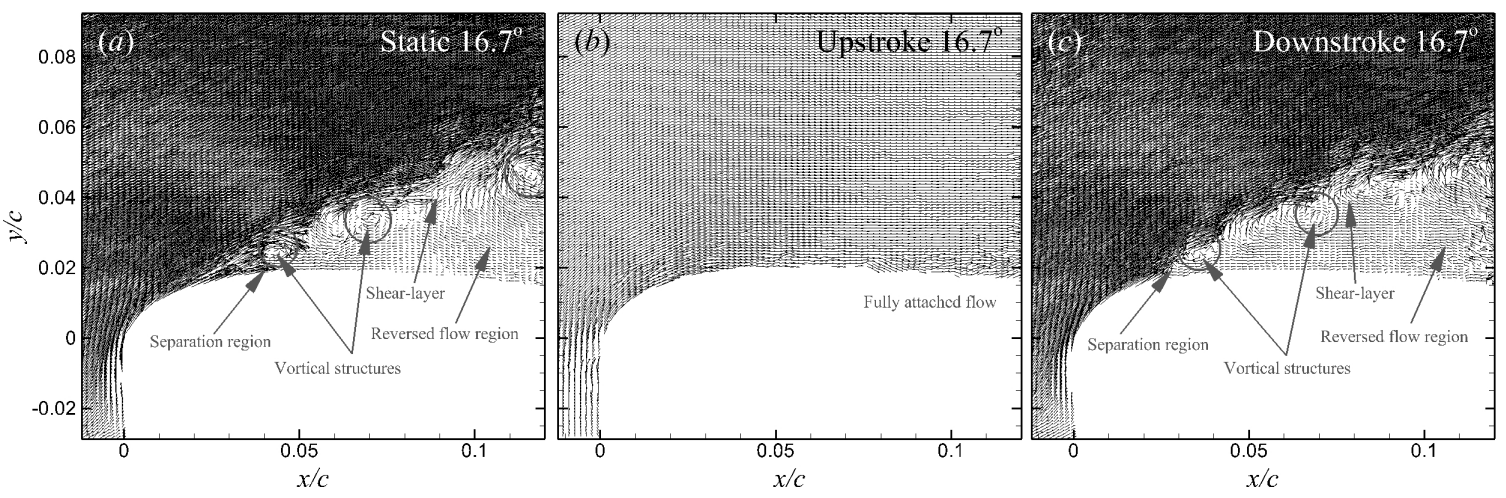

Fig. 8: Instantaneous flow-field structure in the leading edge region of a generic angle of attack above static stall angle. (a) static stall (b) upstroke instant with the flow being delayed above static stall angle $(c)$ downstroke image with separation of the flow-field evident

of stall over the upper-surface.

Furthermore, knowing that dynamic stall is an unsteady phenomenon, and using the 700 images acquired at each test point, the instantaneous structure can be examined to determine if any significant differences exist at a particular test point. The $\alpha=16.7^{\circ}$ static case is well above the static stall angle ( $c f$ figure 5 ) but there is a wide variety of structure as seen in figure 9 . The vast majority of images are highly separated as seen in figure 9 part $(b)$ and $(c)$, but there are instants where the flow-field is fully attached to the surface as seen in part $(a)$. Subtle differences are evident even in the separated images such as part (b) seems to be an intermediate separation, whereas part $(c)$ displays a two part separation with a small vortex located near the leading-edge coupled with a much larger vortex which extends outside the field-of-view.

The $\alpha=22.4^{\circ}$ upstroke also displays a wide variety of structure. Figure 10 part $(a)$ is completely attached within the field-of-view, but instabilities seem to be beginning to form. This wave like motion of the vectors near the surface seems to indicate that a region of separated flow is present outside of the field-of-view and the 

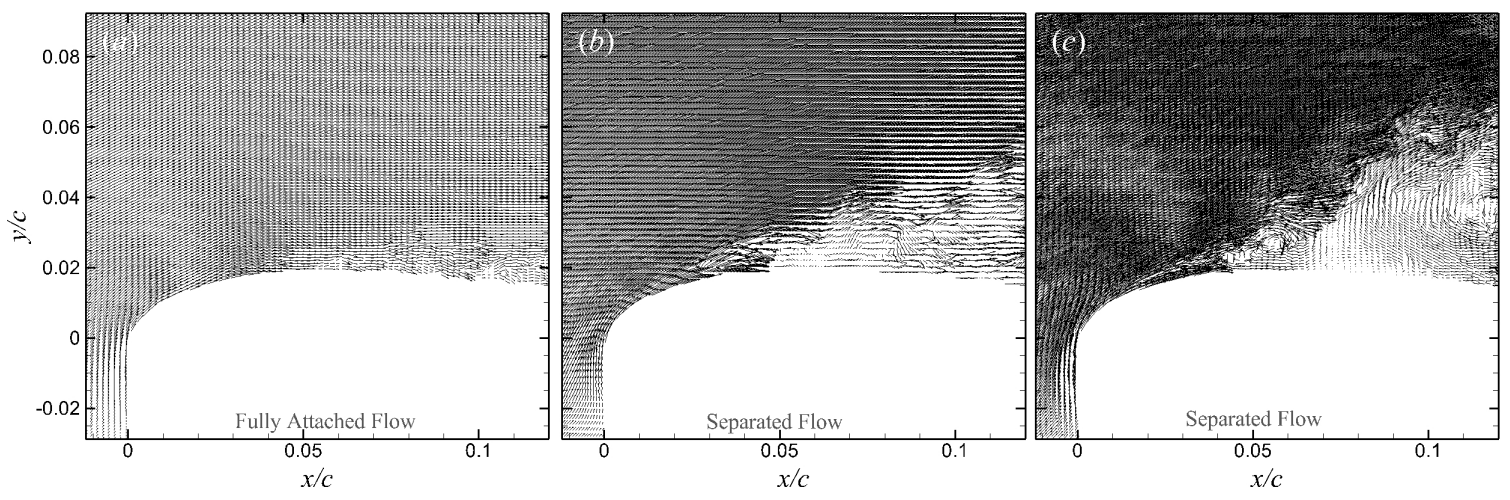

Fig. 9: Instantaneous flow-field structure in the leading edge region. $\alpha=16.7^{\circ}$ static with (a) showing an instant with the flow fully attached, (b) complicated separated flow with few distinct features, and $(c)$ separated flow with dominant visible vortical structures.

effect is propagating upstream disturbing the stream-wise oriented flow. Figure 10 part $(b)$ displays the shear-layer separated from the surface, but no cohesive vortex structures are evident. The final instant figure 10 part $(c)$ represents massive flow separation with a vortical structure equivalent to about $0.08 c$. The large structure seems to be entraining momentum from the free-stream in order to maintain the strength of the vortex.
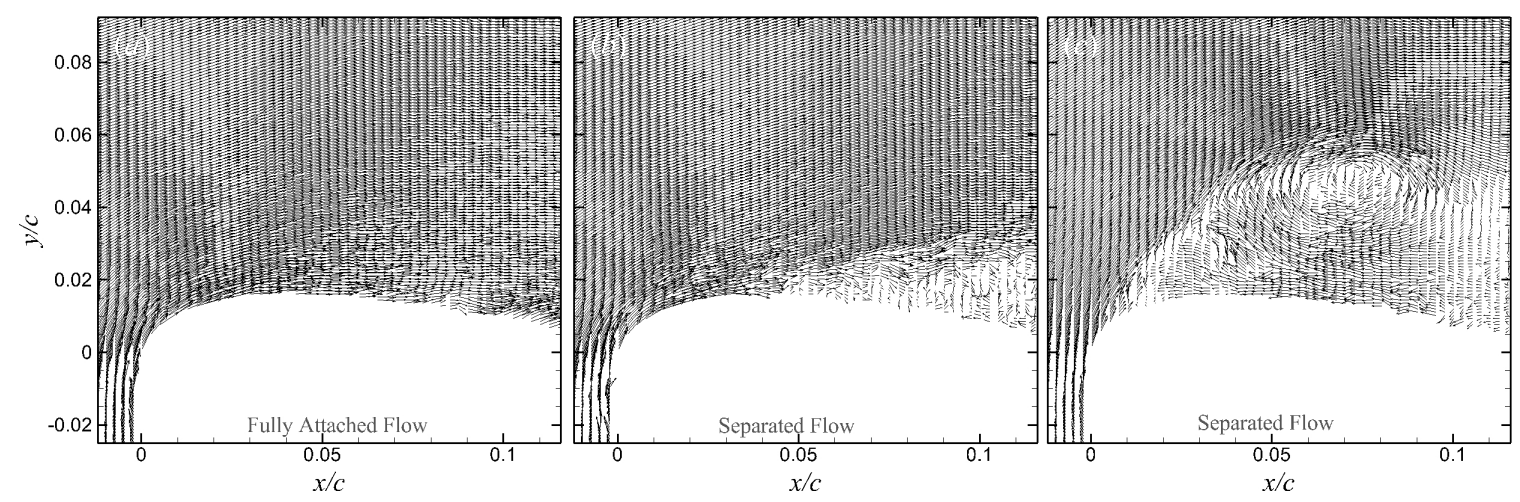

Fig. 10: Instantaneous flow-field structure in the leading edge region. $\alpha=22.4^{\circ}$ upstroke case with $(a)$ showing an instant with the flow fully attached, $(b)$ complicated separated flow with few distinct features, and $(c)$ separated flow with dominant visible vortical structures. 
From the instantaneous shot, the static and downstroke configuration share similarities, but a thorough statistical analyses will demonstrate that there are also some notable differences between the flow-fields.

\section{B. Time-Averaged Properties}

To provide a more statistical description, figures 11-13 depict contour maps of the time-averaged properties for the $\alpha=16.7^{\circ}$ static, upstroke, and downstroke configurations, respectively. Figure parts $(a-d)$ represent the normalized streamwise velocity field, Reynolds-averaged shear stress, streamwise normal stress, and vertical normal stress, respectively. Figure 11(a) displays that the static configuration may be characterized by a large separated flow region, initiating at the leading edge. Within the separation region, significant reversed-flow velocity magnitudes may be observed of up to $5 \%$ of $\mathrm{U}$. Near the leading edge, a relatively large expansion region may also be identified, characterized by increased velocity magnitudes as the approaching fluid accelerates around the leading edge from the stagnation point.

As expected, the Reynolds-averaged normal and shear-stresses can be seen to remain confined to the separated shear-layer. Figure $11(b)$ shows that the negative shear-stress values remained confined to within the shear-layer, near the surface of the airfoil. This is associated with positive streamwise $\left(u^{\prime}>0\right)$ and negative vertical $\left(v^{\prime}<0\right)$ fluctuations and/or negative streamwise $\left(u^{\prime}<0\right)$ and positive vertical $\left(v^{\prime}>0\right)$ fluctuations, and is indicative of the momentum exchange that takes place between the shear-layer and outer inviscid flow, and shear-layer and inner recirculation region. In contrast with the shear-stress component, figure $11(c, d)$ reveals that the normal stresses spread appreciably along the shear-layer with streamwise development. The spread in the normal stress components can be attributed to the movement of the 
shear layer in a "flapping" motion, which will be further described in section D. In general, it can be said that the largest Reynolds stress magnitudes are located near the airfoil surface.

The corresponding upstroke configuration is shown in figure 12, which appears dramatically different from its static configuration counterpart. The primary difference is the flow-field appears to be fully attached for the upstroke. Due to the shear-layer being attached to the surface, the fluid is thought to experience a higher acceleration around the leading edge than for the static case. This leads to a maximum velocity ratio of approximately $2.75 U$ ( $\mathrm{cf} \mathrm{O}(\mathrm{U})$ for the static configuration). This difference is attributed to the position of the shear-layer, which essentially creates an obstacle in the static case, which in turn, reduces the radius of curvature that the outer fluid must follow, thereby reducing the maximum velocity magnitude. The attachment of the shear layer is also evident in the shear and normal stress components. The shear stress, figure $12(b)$, shows interactions only in the leading edge region prior to where the static case shear-layer separated from the airfoil surface. Furthermore, the absence of perturbations over the majority of the field-of-view, coupled with mild interactions, part $(c, d)$, at the edge of the field-of-view, may suggest flow reversal farther downstream, outside the current field-of-view.

Figure 13 shows the corresponding time-averaged velocity field and turbulence statistics of the downstroke configuration. In the contour plots, it can be seen that the downstroke configuration shares similarities with the static case. This is not surprising, given that the airfoil is in a downward pitching motion, where stall has already occurred, causing separation along the entire surface of the airfoil. Expectedly, the Reynolds-averaged normal and shear-stresses, figure $13(b-d)$, tend to be confined to within the separated shear-layer. Similar to the static case, the broad region of significant normal stress seems to indicate movement of the shear-layer in a 

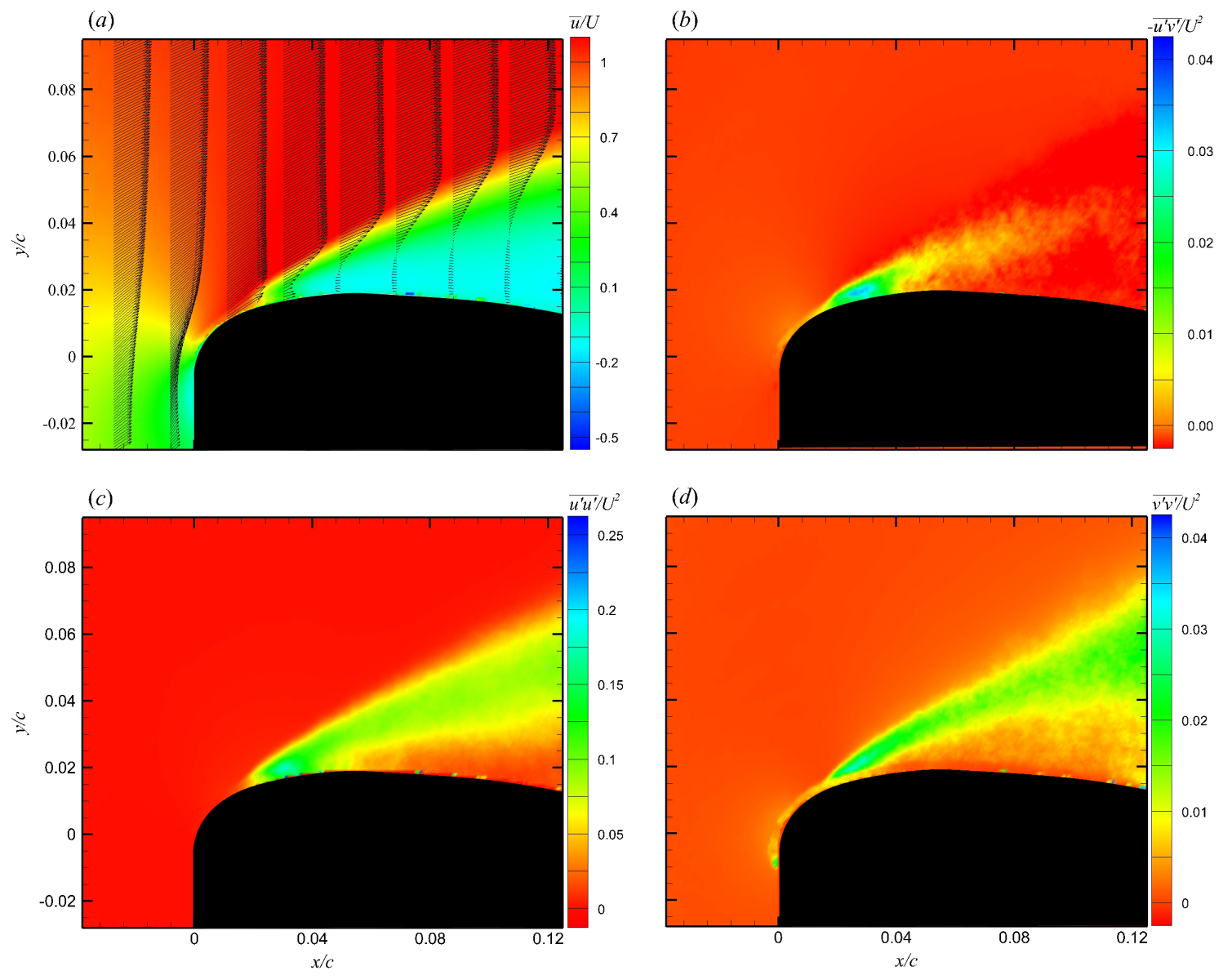

Fig. 11: Time-averaged properties of the NACA 0012 airfoil at a static angle-of-attack $\alpha=16.7^{\circ}$. (a) Mean velocity $\bar{u} / U,(b)$ Reynolds-averaged shear-stress $\overline{u^{\prime} v^{\prime}} / U^{2},(c)$ Reynolds-averaged streamwise normal stress $\overline{u^{\prime} u^{\prime}} / U^{2},(d)$ Reynolds-averaged transverse normal stress $\overline{v^{\prime} v^{\prime}} / U^{2}$. 

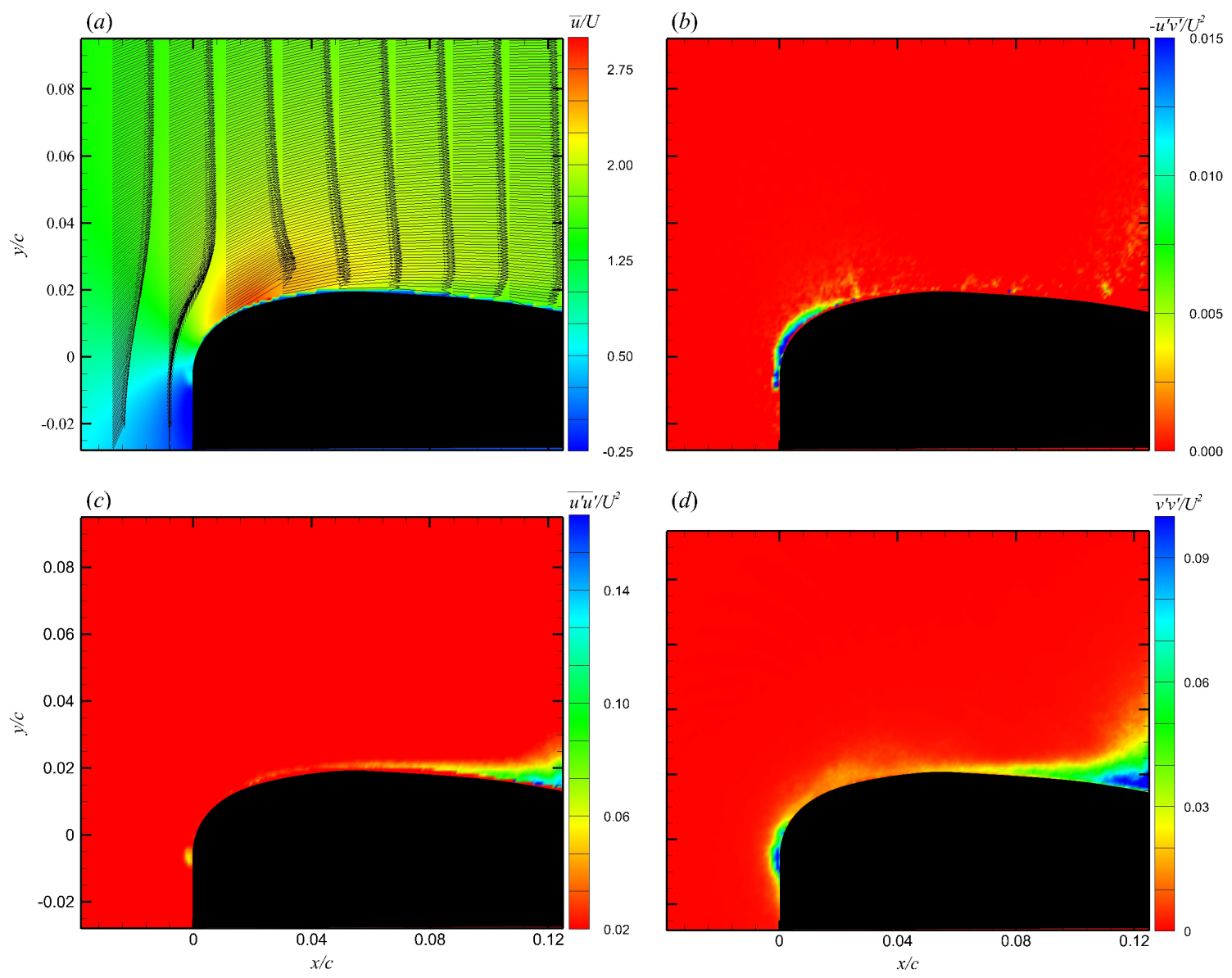

Fig. 12: Time-averaged properties of the NACA 0012 airfoil in the upstroke configuration at an angle-of-attack $\alpha=16.7^{\circ}$. (a) Mean velocity $\bar{u} / U,(b)$ Reynolds-averaged shear-stress $\overline{u^{\prime} v^{\prime}} / U^{2},(c)$ Reynolds-averaged streamwise normal stress $\overline{u^{\prime} u^{\prime}} / U^{2},(d)$ Reynolds-averaged transverse normal stress $\overline{v^{\prime} v^{\prime}} / U^{2}$. 

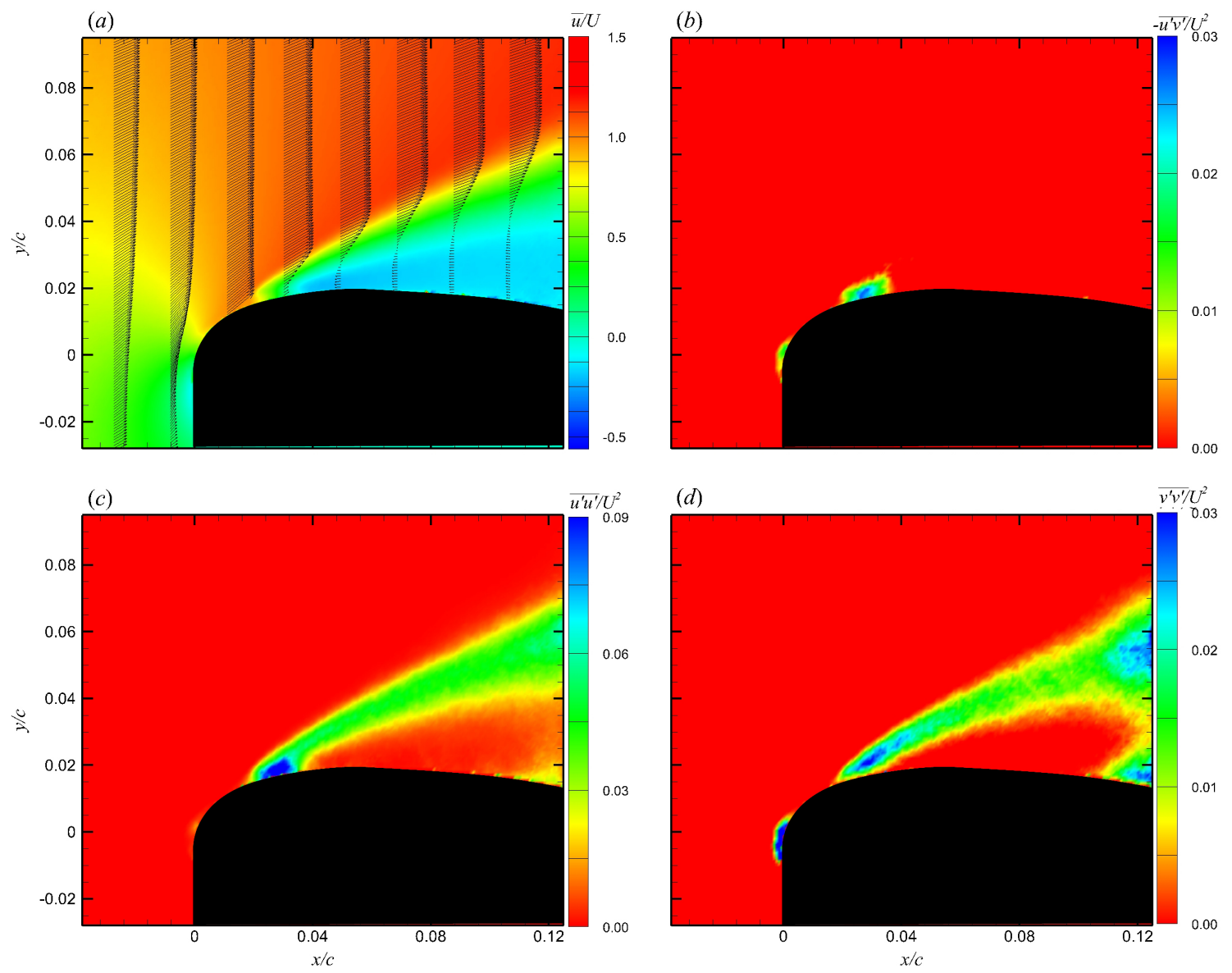

Fig. 13: Time-averaged properties of the NACA 0012 airfoil at static angle-of-attack $\alpha=16.7^{\circ}$. (a) Mean velocity $\bar{u} / U,(b)$ Reynolds-averaged shear-stress $\overline{u^{\prime} v^{\prime}} / U^{2},(c)$ Reynolds-averaged streamwise normal stress $\overline{u^{\prime} u^{\prime}} / U^{2},(d)$ Reynolds-averaged transverse normal stress $\overline{v^{\prime} v^{\prime}} / U^{2}$. 

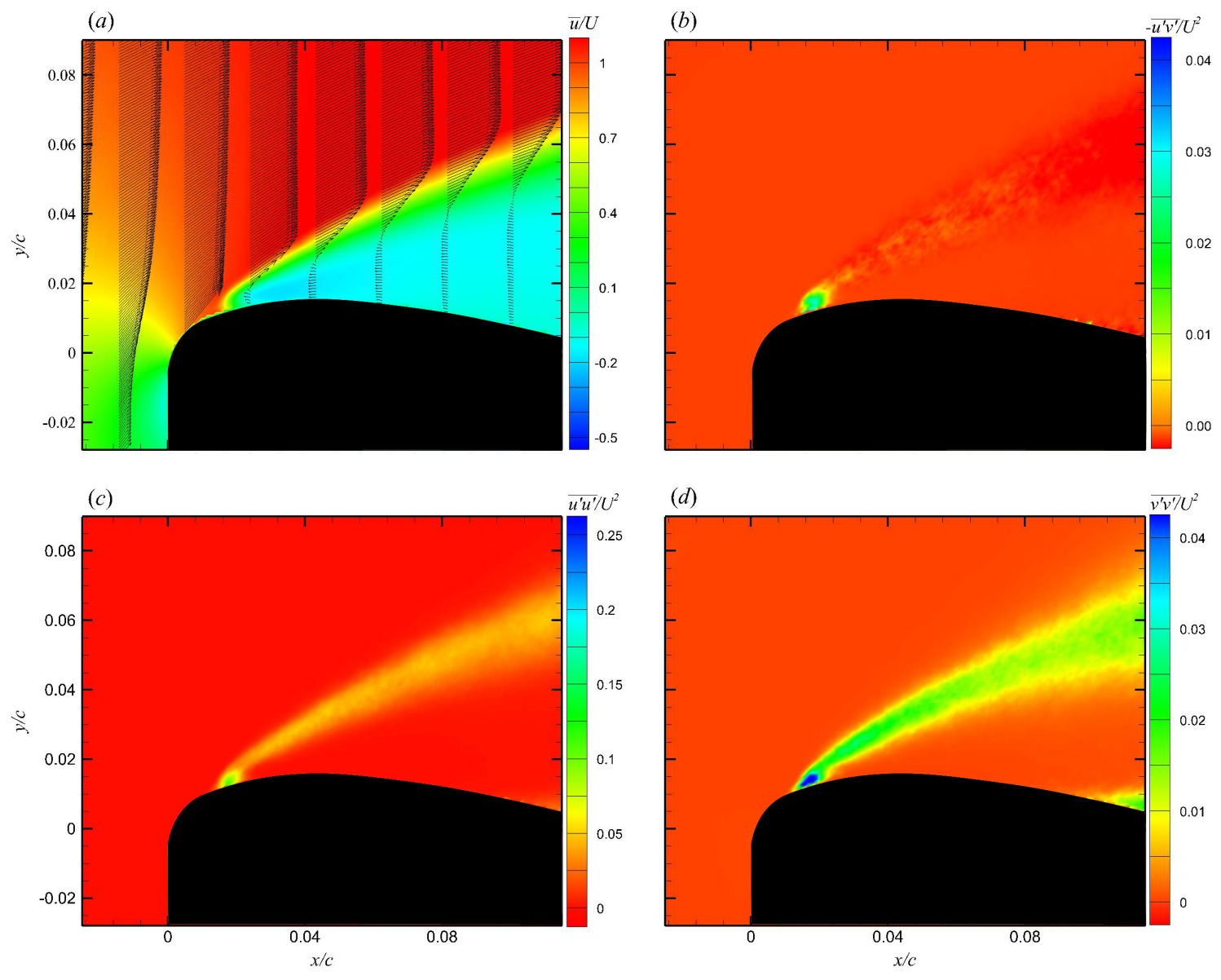

Fig. 14: Time-averaged properties of the NACA 0012 airfoil at a static angle-of-attack $\alpha=22.4^{\circ}$. (a) Mean velocity $\bar{u} / U$, (b) Reynolds-averaged shear-stress $\overline{u^{\prime} v^{\prime}} / U^{2},(c)$ Reynolds-averaged streamwise normal stress $\overline{u^{\prime} u^{\prime}} / U^{2},(d)$ Reynolds-averaged transverse normal stress $\overline{v^{\prime} v^{\prime}} / U^{2}$. 

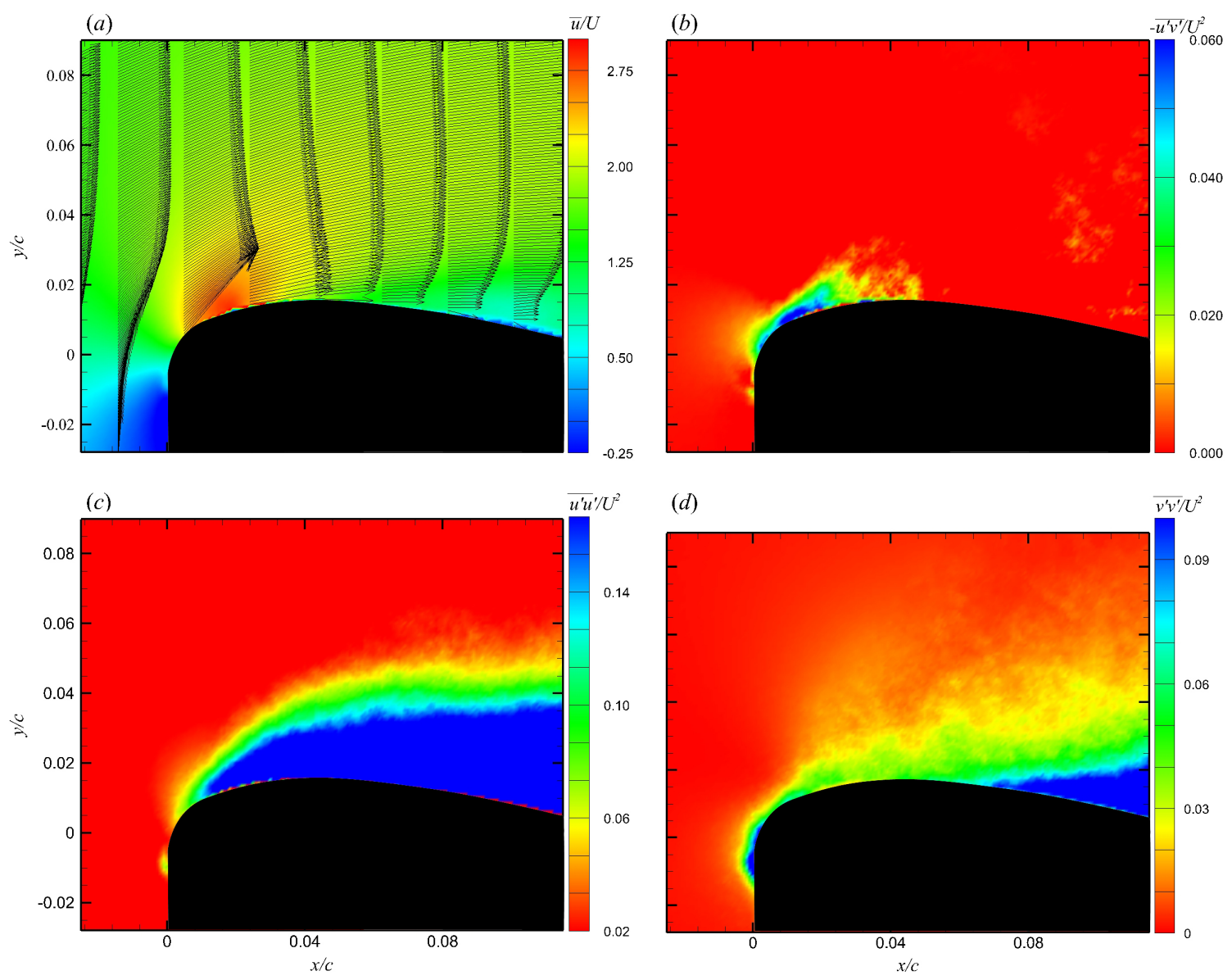

Fig. 15: Time-averaged properties of the NACA 0012 airfoil in the upstroke configuration at an angle-of-attack $\alpha=22.4^{\circ}$. (a) Mean velocity $\bar{u} / U$, (b) Reynolds-averaged shear-stress $\overline{u^{\prime} v^{\prime}} / U^{2},(c)$ Reynolds-averaged streamwise normal stress $\overline{u^{\prime} u^{\prime}} / U^{2},(d)$ Reynolds-averaged transverse normal stress $\overline{v^{\prime} v^{\prime}} / U^{2}$. 

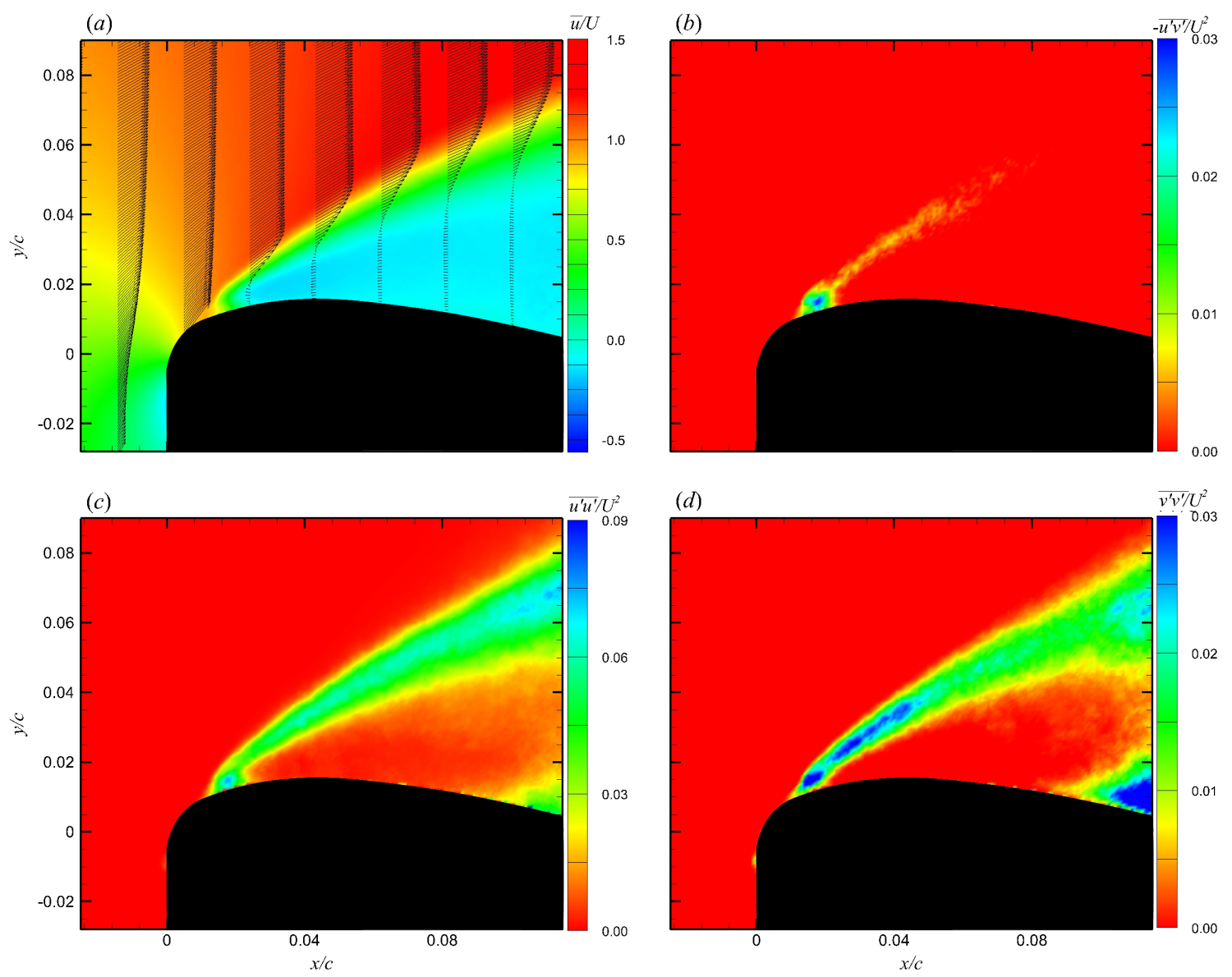

Fig. 16: Time-averaged properties of the NACA 0012 airfoil in the downstroke configuration at an angle-of-attack $\alpha=22.4^{\circ}$. (a) Mean velocity $\bar{u} / U,(b)$ Reynolds-averaged shear-stress $\overline{u^{\prime} v^{\prime}} / U^{2},(c)$ Reynolds-averaged streamwise normal stress $\overline{u^{\prime} u^{\prime}} / U^{2},(d)$ Reynolds-averaged transverse normal stress $\overline{v^{\prime} v^{\prime}} / U^{2}$. 
time-averaged sense. As observed in the static configuration, the expansion around the leading edge appears restricted, which constrains the maximum velocity to be similar to the free-stream velocity.

The $\alpha=22.4^{\circ}$ static case is displayed in figure 14 . As expected by figure 5 the $\alpha=22.4^{\circ}$ results are very similar to the $\alpha=16.7^{\circ}$ case. Large scale separation is evident in the mean, and the Reynolds stresses are localized to the shear-layer.

The $\alpha=22.4^{\circ}$ upstroke case is shown in figure 15, which indicates that flow remains attached on the mean. However, the streamwise normal stress, figure 15 part (c), extends over an especially broad region, indicating distinctly different shear-layer dynamics than the $\alpha=16.7^{\circ}$ case. The distribution of streamwise stress seems to suggest that the flow experiences intermittent separation all the way to the leading edge. This intermittency also would suggest that the $\alpha=22.4^{\circ}$ upstroke is approaching the limit of the dynamic stall effect. The motion of the separation point along with the shear-layer separating from the surface reinforces the data point projected in figure 5. Additional techniques will be used to quantify the differences presented in the Reynolds-stress plots.

The $\alpha=22.4^{\circ}$ is displayed in figure 16 and is similar to the separated cases seen previously. A large area of reversed flow is seen in the time-averaged mean, and the stresses are relegated to the shear-layer. There are subtle differences between the static and downstroke cases, but the differences are subtle which was the motivation behind conditionally averaging the velocity fields to determine any physical differences. 


\section{Statistical Analysis}

To quantify the statements made above, a statistical analysis of the separation point and shear-layer is carried out. Figure 17 and 18 (left column) show the spatial distribution of the probability of observing reversed-flow $\mathrm{P}(u<0)$ in various test configurations, while figure 17 and 18 (right column) show the corresponding distribution of separation location, $x_{s e p} / c$. Estimation of the separation location is problematic because the classical criterion for steady separation (i.e., a vanishing skin friction coefficient at the surface) is no longer valid in unsteady three-dimensional flow [38]. A surrogate for the unsteady separation location was therefore determined, as the zero-crossing of streamwise velocity two rows of vectors $(0.75 \mathrm{~mm})$ from the surface. Using this definition, the separation location is known to within $\pm 0.05 \mathrm{~mm}$ due to the discrete nature of the data. Changes in the threshold used to determine the separation location were negligible up to five vectors from the surface. In all cases, a contiguous region of reversed flow, beginning at the edge of the field of view, must be present on streamwise-oriented flow for the vector to be considered as the separation location. In addition, only the upper surface is considered so that negative $u$ velocities encountered when the flow accelerates around the leading edge from the stagnation point are not considered separation locations.

Under static conditions in figure 17(top row), the separation point tends to oscillate around the mean value of $0.04 x / c$, but occasionally, moves significantly downstream, in some instants so much that the shear-layer collapses onto the surface of the airfoil, eliminating stall within the field-of-view. This phenomenon is evidenced by the values in the right-hand tail of the probability density function, and consistent with the fully attached realizations seen in figure 9. The relatively small number of realizations in this region represents approximately $10 \%$ of the data set when com- 
bined, which is consistent with an airfoil experiencing large-scale separation at this angle of attack in the static configuration. Considering the six primary peaks from figure $17(b)$, with each bin containing at least $3 \%$ of the data set, the separation point appears to wander across approximately $3 \%$ of the chord. Furthermore, the banding of the contour map indicates the region over which the shear layer seems to move in response to the motion of the separation point.

Figure 17(middle row) shows the statistical analysis of the upstroke configuration. From figure 17(c) there appears to be no separation on the upper surface of the wing, which is to be expected for a wing experiencing stall delay. The reversed flow that appears is in less than $1 \%$ of the data set, and is seen at the far edge of the field of view being used for this experiment. The statistical analysis seems to also substantiate the results found in the mean flow data in figure 12 .

Figure 17(bottom row) depicts the downstroke configuration, which portrays a similar probability distribution of reversed flow compared with the static condition. However, figure $17(f)$ suggests that the separation point has a stronger preference for remaining near the mean separation point than the static case. The deviations from the mean of $0.03 x / c$ are approximately \pm 0.01 . While this is comparable to the static configuration for the majority of the data, there is practically no data in the right tail. This is consistent with the time-averaged properties, which show significantly less movement of the shear-layer when compared with the separated static configuration.

Figure 18 (top row) seems to contain characteristics very similar to the $\alpha=16.7^{\circ}$ downstroke, figure 17 (bottom row), rather than the $\alpha=16.7^{\circ}$ static, figure 17 (top row), which was characterized by minimal separation location and reduced shear-layer motion. As expected this occurs for both the pdf and the histogram and elucidates the fact that as the angle-of-attack increases the separation behavior becomes more steady. 

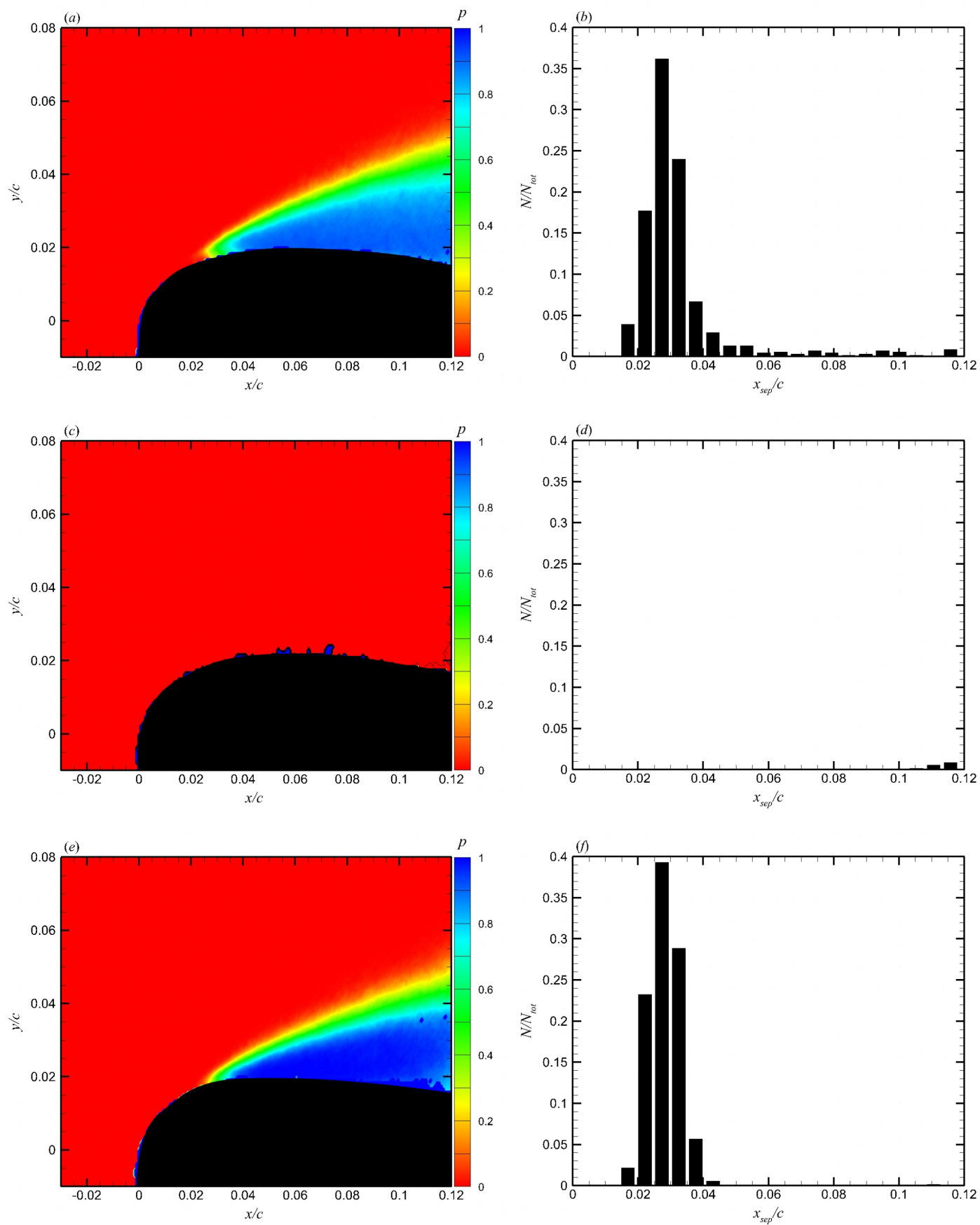

Fig. 17: Statistical analysis of the separated flow region. (left) Probability density distribution of reversed flow $P(u<0)$, (right) probability density function of separation location $x_{\text {sep }} / c$. Results show (top row) $\alpha=16.7^{\circ}$ static, (middle row) $\alpha=16.7^{\circ}$ upstroke, (bottom row) $\alpha=16.7^{\circ}$ downstroke. 

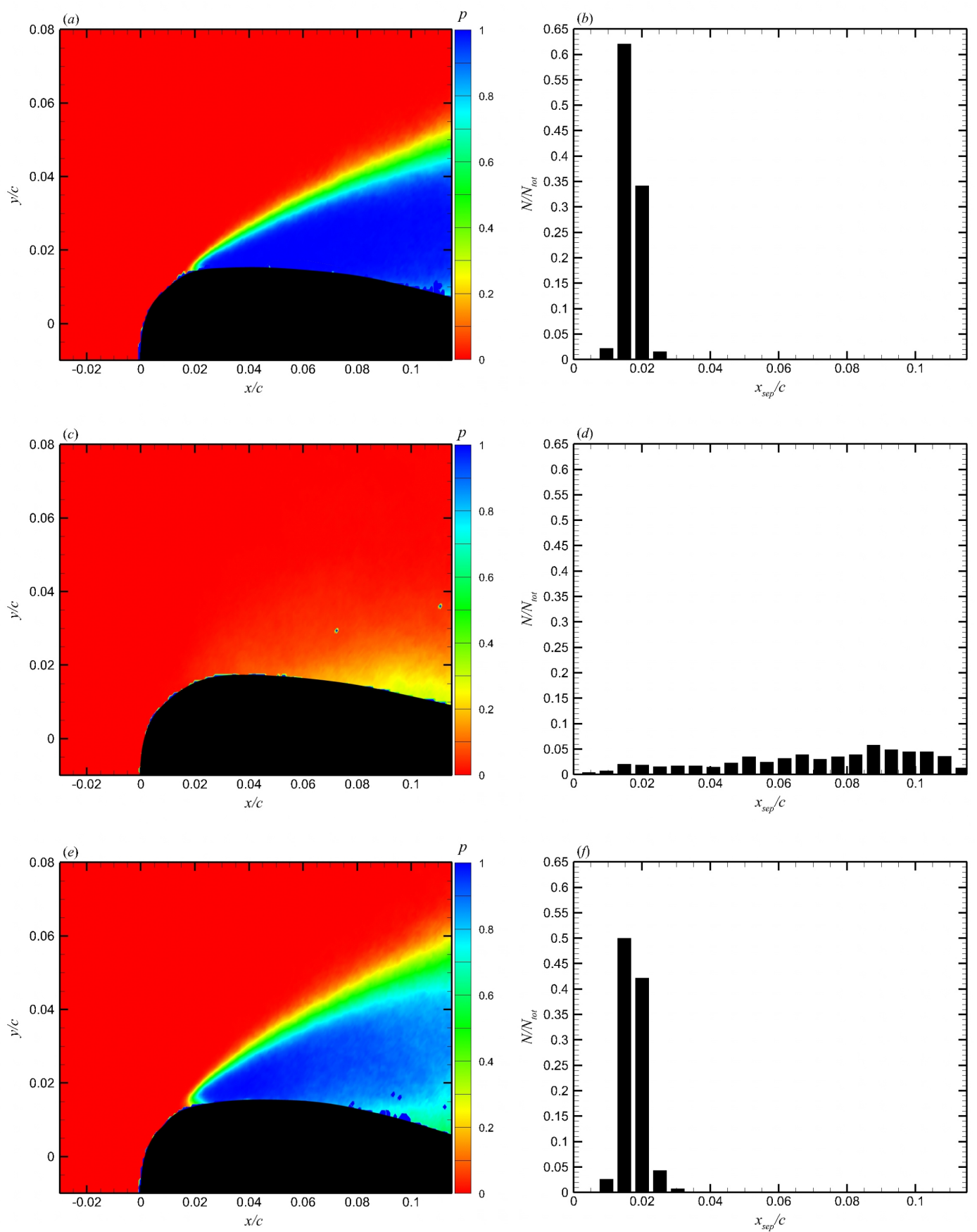

Fig. 18: Statistical analysis of the separated flow region. (left) Probability density distribution of reversed flow $P(u<0)$, (right) probability density function of separation location $x_{\text {sep }} / c$. Results show (top row) $\alpha=22.4^{\circ}$ static, (middle row) $\alpha=22.4^{\circ}$ upstroke, (bottom row) $\alpha=22.4^{\circ}$ downstroke. 
The $\alpha=22.4^{\circ}$ upstroke shown in figure 18(middle row) has unique characteristics, substantially different than the $\alpha=16.7^{\circ}$ upstroke. Figure 18(c) has maximum flow reversal occurring in $30 \%$ of the data set within the field-of-view. The histogram also shows that the movement of the separation point extends across the entire field-of-view, which explains the broad expanse of normal stresses observed in figure $15(b, c)$. The movement actually appears to be truncated indicated by a disproportionate amount of data biased to $x / c=0.12$. This would indicate that if a larger field-of-view was used the the range of motion of the separation point would likely be higher. Thus, the upstroke has realizations of completely attached flow, similar to the $\alpha=16.7^{\circ}$ upstroke case, contrasted against characteristics similar to the static $\alpha=16.7^{\circ}$ case which is fully separated. Statistically, the airfoil is attached in the field-of-view, which agrees with the lift curve data from figure 5 .

The data in figure 18 also indicates minimal shear-layer and separation point motion. Therefore there is little to differentiate the static and downstroke flow-field characteristics in the $\alpha=22.4^{\circ}$ case as opposed to significant differences evident in the $\alpha=16.7^{\circ}$ configurations. These differences will be developed further in section D.

The three states of the $\alpha=16.7^{\circ}$ shown in figure 17 continue the traditional results established by figures 11-13 where the static and downstroke contain no separation while the upstroke shows no indications of a stalled airfoil. This is contrasted against the results displayed in figure 18 which are similar for the static and downstroke, but differ significantly for the upstroke configuration. This continues the idea that the $\alpha=22.4^{\circ}$ case is on the edge of the dynamic stall effect for the NACA 0012 airfoil at these conditions. 


\section{Conditionally Averaged Velocity Fields}

To further highlight the differences between the static and downstroke configurations, conditional averaging is implemented. A detailed description on how this procedure is implemented can be found in Chapter III section D. Figure 19(a,b) displays the perturbation fields for the $\alpha=16.7^{\circ}$ static configuration. The largest perturbations occur in the separation point region. Significant perturbations also appear in the shear-layer, which reinforces the notion that the shear-layer undergoes an unsteady flapping motion in the static configuration.

Figure 19(c,d) represents the downstroke configuration, which is noticeably different from the static counterpart. The perturbations appear limited to a small region close to the time-averaged separation location. This is substantiated by the data in figure $17(e, f)$, which indicate a more stable shear-layer and separation location. Comparing the figure 19 plots, the static case $(a, b)$ exhibits a relatively unstable separation location and shear-layer. In contrast, the downstroke $(c, d)$ has an unstable separation location, but the shear-layer appears steadier. The reason why the shear-layer is more stable during the downstroke case compared to the static can be found at the end of section $\mathrm{E}$ after the complexities of the leading edge vortex have been discussed, since this phenomenon plays an important role in accounting for this disparity.

\section{E. Phase-Averaged Representation}

A detailed motivation and description for the process of phase averaging can be found in chapter III section E. To use this procedure, a representative reference point must be selected for the analysis. In the present study, a point in the external flow was chosen, which is consistent with the choice of others [39]. Different reference locations in the free stream give similar results, as shown in figure 20 , which displays 

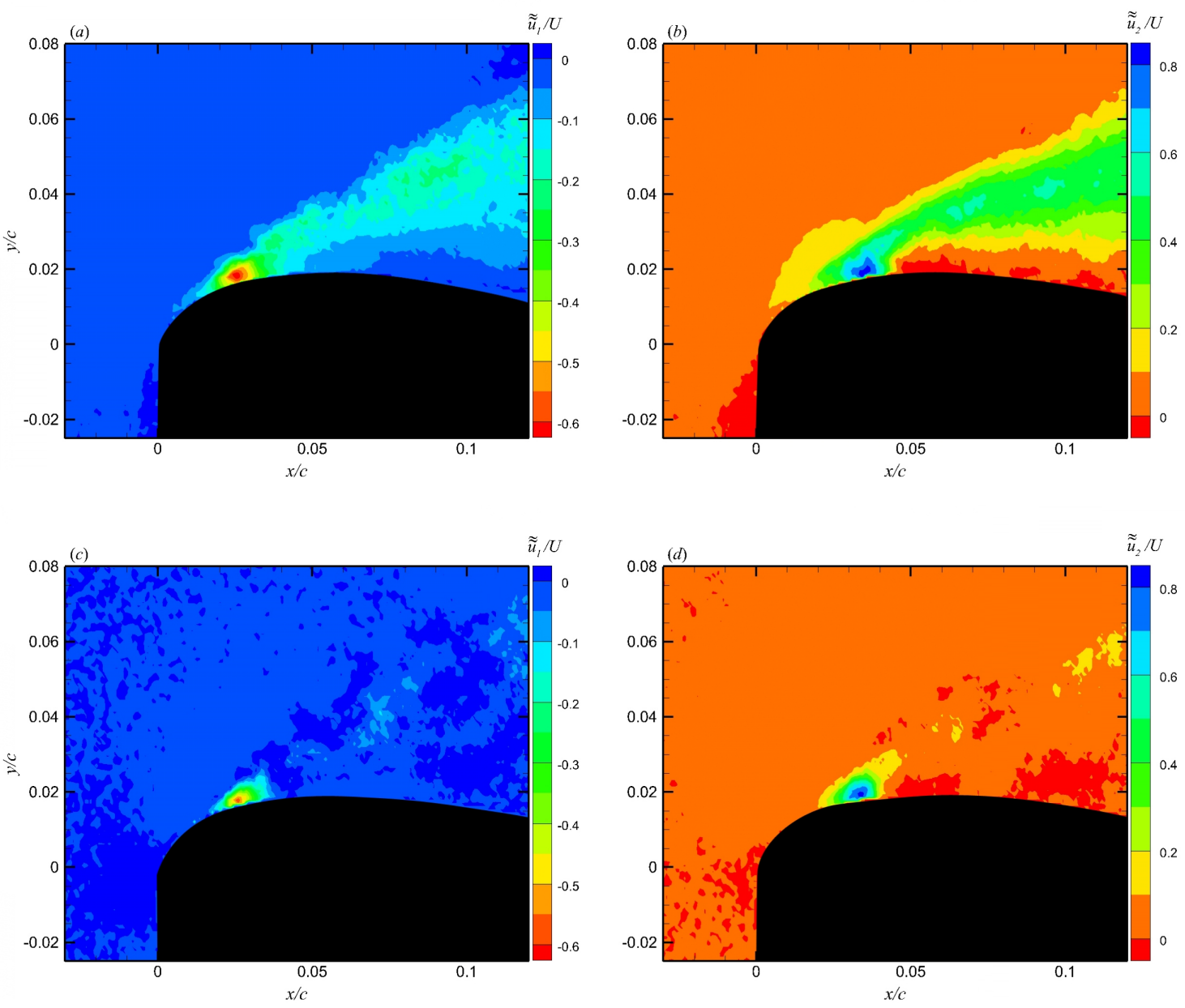

Fig. 19: Conditional averaged perturbation fields for the $\alpha=16.7^{\circ}$ static (top row) and downstroke (bottom row) configuration. (a,c) Conditional average of upstream separation, $(b, d)$ conditional average of downstream separation. Note both fields are mean subtracted. 
the power spectral density(PSD) on the left, and the Hilbert phasor diagram on the right. This phasor diagram represents the dynamic stall cycle as a rotating vector, or sweeping arm of the Hilbert amplitude $a(t)$ and the phase $\phi(t)$. One key difference between the phasor plots is that the characteristics seem to shift around the plot based on the location of the point selected. This is mainly due to the points farther downstream feel the effect at a later time due to the convection of the characteristics. This enables the entire dynamical stall process to be visualized in a rather novel coordinate system, with the added benefit of visualizing the complete phase space composed of numerous wing oscillations.

Figure 20(c,d), displays the point chosen for analysis, but the analysis holds for any free-stream point. Note the large spectral peak at $k \approx 0.1$ associated with the fundamental frequency of the wing motion. Additional higer-order and subharmonics are also observed.

The phase diagram reveals several interesting features, characterized by three distinct lobes. Since the phase angle is arbitrary, it was shifted to coincide with the phase of the wing. Therefore, the phase region $\phi=0^{\circ}-180^{\circ}$ corresponds to the upstroke motion, whereas the phase region $\phi=180^{\circ}-360^{\circ}$ corresponds to the downstroke motion. The lobe that occurs at $\phi=60^{\circ}$ is where the airfoil is experiences the extension of quasi-linear lift beyond the static stall angle. The lobe that occurs between $\phi=120^{\circ}-150^{\circ}$ appears to be associated with the evolution of the leading edge vortex. The response of the Hilbert amplitude can be traced to the decrease in convex curvature of streamlines in the outer flow, which causes an appreciable increase in the vertical velocity and hence amplitude. The lobe that occurs between $\phi=180^{\circ}-250^{\circ}$ seems to be the result of the shear-layer adjusting to the change in wing position. Here, the wing has terminated its upstroke motion, and begins the downstroke. This change in wing motion causes the shear-layer to be momentarily 

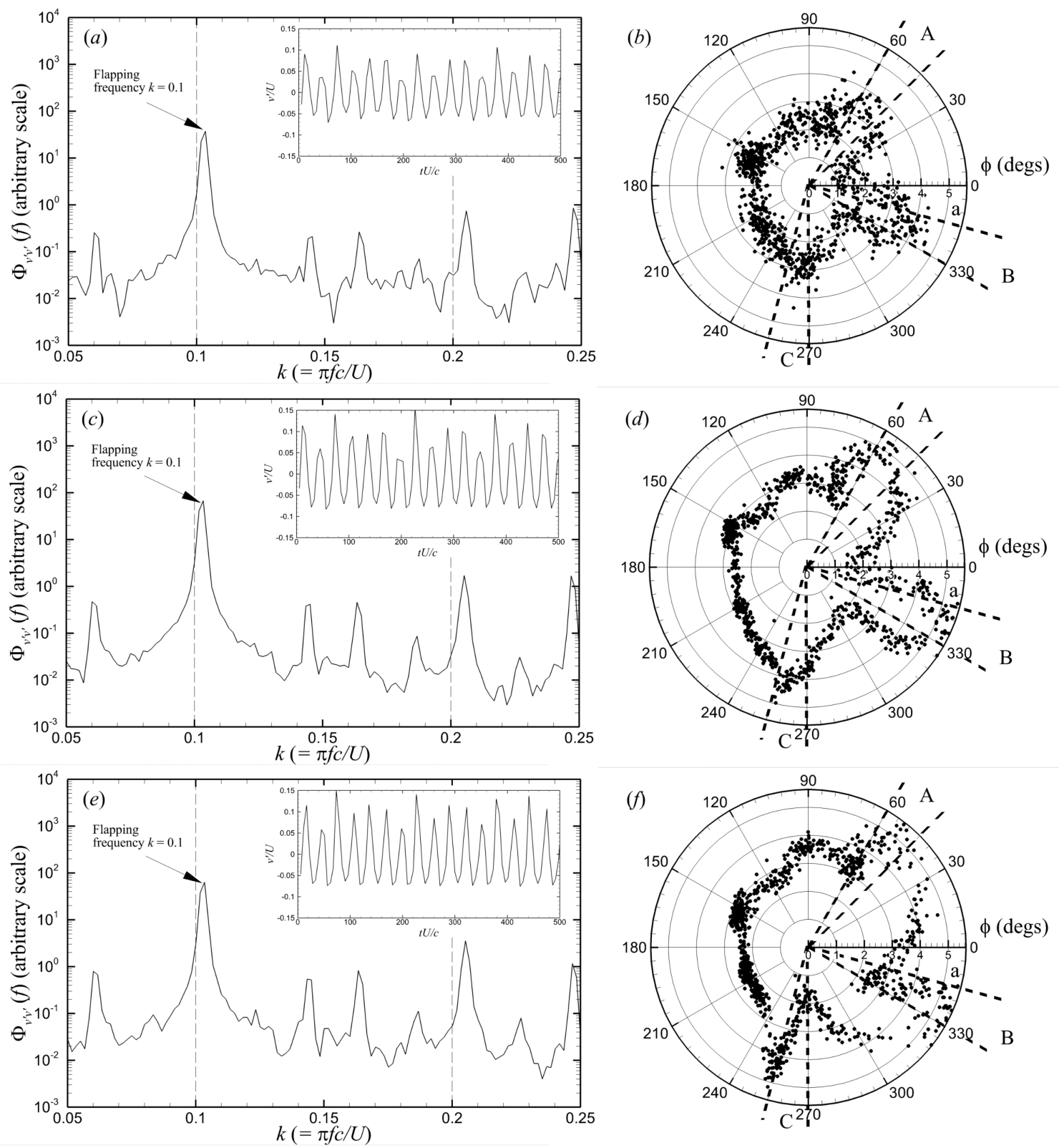

Fig. 20: Spectral and Hilbert transform analysis. The three rows represent different reference locations within the free-stream where the Hilbert transform was computed. (Left column) Power spectral density of the reference signal $v(t)$, with original signal inset. (Right column) Hilbert phasor diagram. Measurement locations: top row ($0.027 x / c, 0.089 \mathrm{y} / \mathrm{c})$, middle row $(0.00 \mathrm{x} / \mathrm{c}, 0.069 \mathrm{y} / \mathrm{c})$, and bottom row $(0.022 x / c$, $0.085 \mathrm{y} / \mathrm{c}$ ) referenced to $\alpha=16.7^{\circ}$ coordinate system. 
suspended while the wing begins its downward motion. This suspension manifests as an increase of the Hilbert amplitude and thus results in a peak in the phasor diagram. Note that numerous wing oscillation cycles are shown in the phasor diagram, and that despite its complexity there appears to be relatively little cycle-to-cycle variation in the phase-averaged sense.

\section{F. Phase Averaging Morphology}

Since each point in phase represents an instantaneous snapshot, it may be combined with images at a similar phase to create phase-averaged realizations. Several of these are depicted in figure 21. These images were created by dividing the phase portrait into 24 equal segments constituting approximately $1.5^{\circ}$ change in angle of attack per segment. These values represent a compromise between obtaining enough phase segments to represent the physics of motion, while still maintaining a sufficient number of images within each segment to enable a meaningful average. For the purposes of discussion, only a select number of phase averages are shown, judiciously chosen to represent the complete cycle. The following description helps extend the classical description of the dynamic stall process, specifically related to the formation and evolution of the leading edge vortex.

Figure 21(a) shows the upstroke motion of the airfoil beyond the static stall angle. Although it cannot be observed in the present field-of-view, flow reversal will develop from the trailing edge, and work its way towards the leading edge [2]. By figure 21(b), the boundary layer has now detached from the surface of the airfoil. The instantaneous vorticity distributions show clockwise-rotating vortical structures developing in the shear-layer. A relatively small vortex is created in the process, which is momentarily suspended over the leading edge region in the phase-averaged sense. 
Although this vortex is quite small, being of the order of the shear-layer thickness, it is present in several other instantaneous images at the same phase, and is therefore not considered to be an artifact. In reality, several similarly-sized vortices are observed to be present in the shear-layer. With further streamwise development, the shearlayer then rolls up into what is known as the dynamic stall vortex, or leading edge $\operatorname{vortex}(\mathrm{LEV})$.

With further increase in angle-of-attack, the leading edge vortex grows rapidly, entraining momentum from the shear-layer in order to sustain this process, as seen in figure $21(c)$. This is consistent with the development of the shear-layer KelvinHelmholtz instability, which causes vortical structures to grow, as well as the merging of clockwise-rotating vortices into larger ones, although visual evidence of vorticies merging were not found in this representation due to the absence of time-resolved data. As the vortex continues to strengthen, it begins shedding vorticity into the area of reversed flow between the wing and the shear-layer, which is displayed in figure $21(d)$. The leading edge vortex seems to be momentarily suspended over the leading edge of the wing, also referred to herein as the primary vortex, while the peripherial vorticity coalesces into a secondary vortex, which begins drawing in fluid from the shear-layer. Mulleners et al. [13], also observed such behavior, and considered it within the contex of vortex formation in the wake behind a cylinder. The primary vortex grows to saturation before being pinched off from the separated boundary layer that provides its circulation. However, due to the nature of the phase-averaging procedure, certain details about the precise formation mechanisms are lost. Nevertheless, it can be seen that both the primary and secondary vortex structures are convected downstream. The primary vortex appears to convect more slowly than the secondary vortex in the phase-averaged sense, because by figure $21(e)$, the latter has moved a significant distance downstream, whereas the primary vortex remains 
in approximately the same location. By figure $21(f)$, the secondary vortex appears almost out of view, whereas the primary vortex appears to weaken, as evidenced by inspection of the vorticity contours (not shown here for brevity). The dynamics could help explain the broadening of the surface pressure signals as observed by others [11]. Consistent with the observations of Ahmed and Chandrasekhara [7], three regions of interest associated with the leading edge vortex may be identified: (1) a region where the dynamic stall vortex forms and gathers strength, (2) a region where it convects along the surface growing at the same time, and (3) a region where the vortex moves rapidly away from the surface and into the external flow.

With further phase development shown in figure $21(\mathrm{~g})$, the wing begins its downstroke journey. This occurs near the phase that the shear-layer appears at its maximum distance from the airfoil surface. At this point, no vortices are visible in the phase averages, just a large region of reversed-flow. An analysis of the instantaneous images shows vortices appearing at seemingly random locations, which when averaged together are smeared into a region of reversed flow. This random distribution of vortices is in contrast to their systematic distribution on the stall-delayed upstroke motion, which is perhaps why they are so concentrated in the phase averages. This lack of general reproducibility during the downstroke, as opposed to the upstroke, has been noted by several authors, and is attributed to the intrinsic characteristics of the dynamical separation process itself, whereby the separated flow is more sensitive to variations of the boundary conditions inherent in the experiment. Hence, the downstroke has more cycle-to-cycle variation than the upstroke or static case [13]. This is not to be confused with the decrease in motion of the shear-layer in the downstroke configuration. Here, we believe the existence of the leading edge vortex plays an important role; through its relatively slow phase evolution it may act as a stabilizing influence on the shear-layer during the downstroke, since it presents itself as an obsta- 
cle the flow must negotiate. Due to an unsteady transfer of momentum upstream near the separation location, the instantaneous separation point still exhibits an unsteady motion, thereby reconciling the disparate separation point and shear-layer motions depicted in figure 17. The absence of the leading edge vortex mechanism in the static case also reconciles the statistical results described earlier in figure $19(a-d)$. This means that the phase-averaging procedure has to be implemented carefully, because it eliminates instantaneous effects and includes these cycle-to-cycle variations in the phase-averaging procedure. As noted by Wernet et al. [15], this makes the comparison between experimental and numerical results particularly difficult. Later in the phase development, the shear layer begins the reattachment process from the leading edge, which is shown in figure 21(h). Given the limited field-of-view, however, it cannot be said whether or not large-scale separation is still present farther downstream [7]. This is especially complicated by the fact that it is known that, unlike steady reattachment, which is a relatively sudden event, the reattachment process is completed over the entire airfoil upper surface over a range of angle of attack when the airfoil oscillates [7]. 

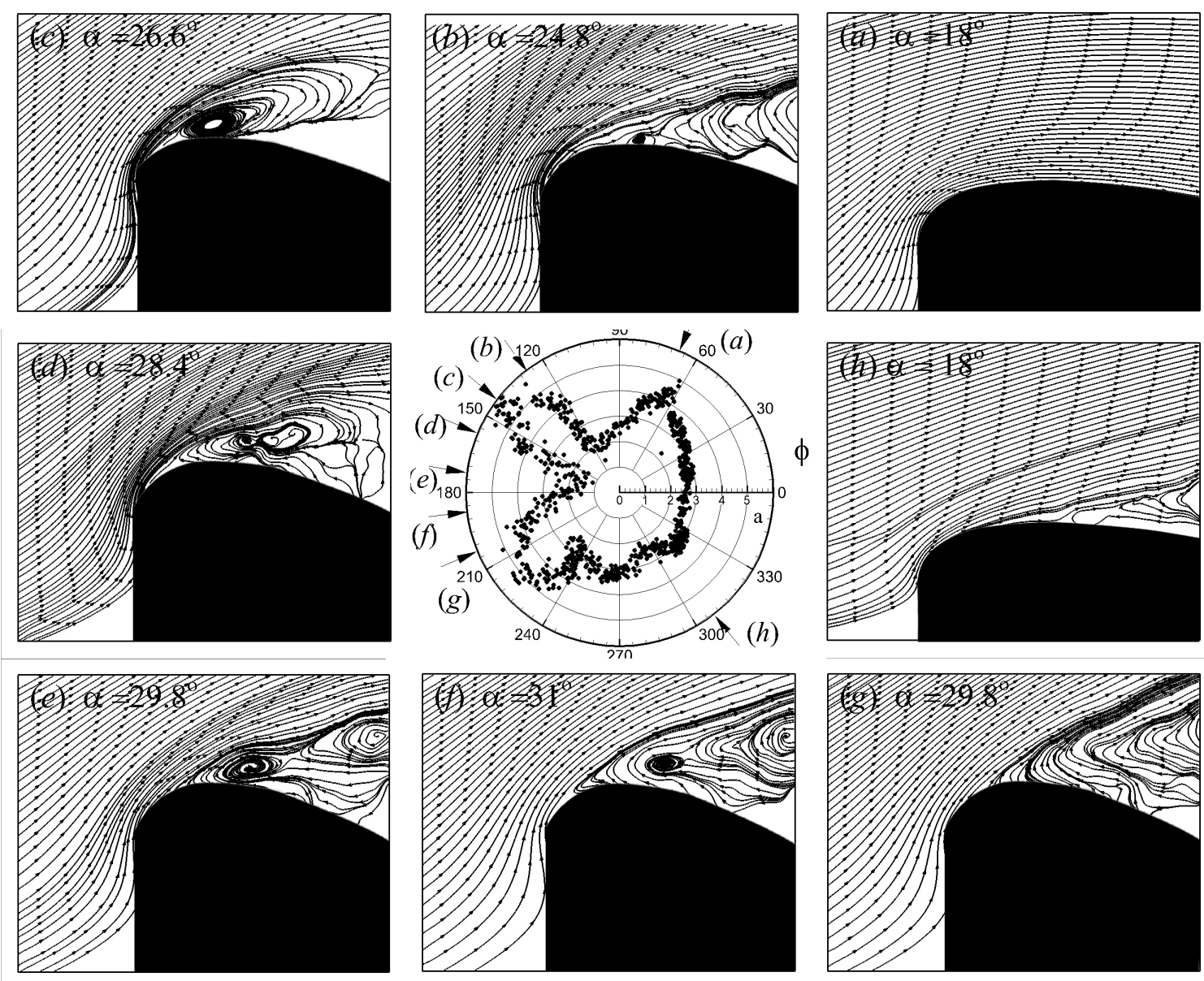

Fig. 21: Selected phase-ensembled realizations showing dynamic stall flow-field evolution displayed with streamlines and velocity vectors at a skip of 1 in 8 in the streamwise direction and 1 in 2 in the transverse direction. Approximate angle-ofattack and phase angle are noted in the figure. Field-of-view is $0.16 c \times 0.1 c$. The airfoil masks are representative and for illustration only. 


\section{CHAPTER V}

\section{CONCLUSIONS \& RECOMMENDATIONS}

\section{A. Summary of Results}

The leading edge flow structure of the NACA 0012 has been experimentally investigated under dynamic stall conditions $\left(M=0.1, \alpha=16.7^{\circ} R e_{c}=1 \times 10^{6}\right)$ using planar PIV. The airfoil was dynamically pitched about the $1 / 4$ chord at a reduced frequency, $k=0.1$. As a result of this work, the following conclusions may be drawn: Under dynamical pitching, both the time-averaged and instantaneous flow-fields differed dramatically from their static counterparts. As expected, during the upstroke the flow remained attached in the leading edge region above the static stall angle, whereas during downstroke, the flow remained separated below the static stall angle. Turbulence statistics were computed and analyzed. Statistical analyses and conditional averaging indicated that the leading edge vortex acts as momentum exchange mechanism and flow obstacle which explains the unstable motion in the separation location in both the static and downstroke, but stabilizes the shear-layer in the downstroke configuration whereas the static shear-layer continues in a more unsteady motion in the absence of the leading edge vortex. A phase averaging procedure involving triple velocity decomposition in combination with the Hilbert transform enabled the entire dynamical stall process to be visualized in a novel coordinate system, with

the added benefit of visualizing the complete phase space composed of multiple wing oscillations. The complex formation and evolution of the leading edge vortex was observed. This vortex was seen to grow, interact with surrounding vorticity, detach from the surface, and convect downsteam. These observations led to a modified physical 
description of the classical idealized picture of dynamic stall. In the present model, the leading edge vortex appears as a coalescence of shear-layer vortices rather than a distinct singular vortex. Only in the phase-averaged sense is the latter description the case. In addition, the leading edge vortex seems to lead to the appearance of a secondary vortex, which significantly increases the chordwise extent of fluid being influenced by the vortical structure.

\section{B. Future Work}

Through the completion of this campaign on dynamic stall in the DSF located at Texas A\&M University, much was learned and hopefully will be used as a guide for any future work in this facility.

The planar particle image velocimetry technique was established and utilized effectively resulting in a field-of-view of $75 \mathrm{~mm} \times 56 \mathrm{~mm}$. Additional attempts were made to shrink the field-of-view, but at best using the current optics available the field-of-view may only be shrunk by an additional 10-15\%. This limitation is mostly due to the focusing constraints since the measurement locations is over a meter away from the camera. Furthermore any smaller field-of-view will increase the effective

jitter of the airfoil in the acquired images. This will require additional post processing to allow for the calculations of means and turbulence statistics.

The facility has recently had an upgrade of motor which will extend the Mach number range to 0.4 and possibly 0.5 . Realistic retreating blade stall occurs in the range of $M=0.2-0.3$, which should be where the follow on campaign should be conducted. The data acquired in this work established the technique and a baseline for future work, but additional information on compressibility effects would be of great use to the current literature. There are only a handful of studies in existence that 
give full-field velocimetry data.

Another possibility is to use stereoscopic particle image velocimetry which will give the third velocity component. Dynamic stall is known to be a highly 3-dimensional problem, which means the conclusions being drawn are limited since the data acquired herein is limited. In order to do stereo, adjustments will need to be made to the current infrastructure so that two cameras can be utilized.

If additional models are constructed for the windtunnel, they should include the ability to acquire surface pressure. One major point lacking in this study was that surface pressure could not be compared to the velocimetry data which would be very useful for further analysis of the current dynamic stall model. Also plugs can be inserted if acquiring surface pressure isn't possible for a given test, but the capability should be included on all future models. 


\section{REFERENCES}

[1] McCroskey, W. J., "Unsteady Airfoils," Annual Review of Fluid Mechanics, Vol. 14, 1982, pp. 285-311.

[2] Carr, L. W., "Progress in analysis and prediction of dynamic stall," Journal of Aircraft, Vol. 25, 1988, pp. 6-17.

[3] Sheng, W., Galbraith, R. A. M., and Coton, F. N., "Return from Airfoil Stall During Ramp-Down Pitching Motions," Journal of Aircraft, Vol. 44, No. 6, 2007, pp. $1856-1864$.

[4] Sheng, W., Galbraith, R. A. M., and Coton, F. N., "Inproved Dynamic-StallOnset Criterion at Low Mach Numbers," Journal of Aircraft, Vol. 44, 2007, pp. $1049-1052$.

[5] Sheng, W., Galbraith, R. A. M., and Coton, F. N., "A new Stall-Onset Criterion for Low Speed Dynamic-Stall," Journal of Solar Energy Engineering, Vol. 128, 2006, pp. 461-471.

[6] Choudhuri, G. and Knight, D., "Effects of compressibility, pitch rate, and Reynolds number on unsteady incipient leading-edge boundary layer separation over a pitching airfoil," Journal of Fluid Mechanics, Vol. 308, 1996, pp. 195-217.

[7] Ahmed, S. and Chandrasekhara, M. S., "Reattachment studies of an oscillating airfoil dynamic stall flowfield," AIAA Journal, Vol. 32, No. 5, 1995, pp. 10061012. 
[8] Choudhuri, P., Knight, D., and Visbal, M. R., "Two-dimensional unsteady leading-edge separation on a pitching airfoil," AIAA Journal, Vol. 32, No. 4, 1994, pp. 673-681.

[9] Crisler, W., Krothapalli, A., and Lourenco, L., "PIV Investigation of High Speed Flow over a Pitching Airfoil," 32nd Aerospaces Sciences Meeting \&6 Exhibit, No. AIAA-94-0533, 1994.

[10] Ekaterinaris, J. A., Chandrasekhara, M. S., and Platzer, M. F., "Recent Developments in Dynamic Stall Measurements, Computations and Control," 43rd AIAA Aerospace Scineces Meeting and Exhibit, No. AIAA-2005-1296, 2005.

[11] McAlister, K. W., Carr, L. W., and McCroskey, W. J., "Dynamic stall experiments on the NACA 0012 airfoil," Tech. rep., NASA, 1978.

[12] McCroskey, W. J. and Pucci, S. L., "Viscous-Inviscid Interaction on Oscillating Airfoils in Subsonic Flow," AIAA Journal, Vol. 20, No. 2, 1982, pp. 167-174.

[13] Mulleners, K., Henning, A., Mai, H., and Raffel, M., "Investigation of the unsteady flow development over a pitching airfoil by means of TR-PIV," 27th AIAA Applied Aerodynamics Conference, No. AIAA 2009-3504, 2009.

[14] Sheng, W., Galbraith, R. A. M., and Coton, F. N., "A Modified Dynamic Stall Model for Low Mach Numbers," 45th AIAA Aerospace Sciences Meeting and Exhibit, No. AIAA-2007-0626, 2007.

[15] Wernert, P., Geissler, W., Kompenhans, J., and Raffel, M., "Experimental and numerical investigations of dynamic stall on a pitching airfoil," AIAA Journal, Vol. 34, No. 5, 1996, pp. 982-989. 
[16] Harris, F. D. and Pruyn, R., "Blade Stall-Half Fact, Half Fiction," Journal of the American Helicopter Society, Vol. 13, No. 2, 1968, pp. 27-48.

[17] Ham, N. and Garelick, M., "Dynamic Stall Considerations in Helicopter Rotors," Journal of the American Helicopter Society, Vol. 13, No. 2, 1968, pp. 49-55.

[18] Ericsson, L. and Reding, J., "Fluid mechanics of dynamic stall part I. Unsteady flow conceptsl," Journal of Fluids and Structures, Vol. 2, No. 1, 1988, pp. 1-33.

[19] Watson, K., Cormey, J., Komerath, N., and Diottavio, J., "Diagnostics of 3D Dynamic Stall on Rotor Blades," Tech. rep., Georgia Institute of Technology, 2008.

[20] Carta, F., "Experimental Investigation of the Unsteady Aerodynamic Characteristics of a NACA 0012 Airfoil," Res. Rep M-1283-1, United Aircraft Corp., 1960.

[21] Liiva, J. and Davenport, F., "Dynamic Stall of Airfoil Sections for High-Speed Rotors," Journal of the American Helicopter Society, Vol. 14, No. 2, 1969, pp. 26 33.

[22] McCroskey, W. and Fisher, R., "Detailed Measurements on a Model Rotor in the Blade Stall Regime," Journal of the American Helicopter Society, Vol. 17, No. 1, 1972, pp. 20-30.

[23] Ericsson, L. and Reding, J., "Dynamic Stall of Helicopter Blades," Journal of the American Helicopter Society, Vol. 17, No. 1, 1972, pp. 11-19.

[24] McCroskey, W., Carr, L. W., and McAlister, K. W., "Dynamic Stall Experiments on Oscillating Airfoils," AIAA Journal, Vol. 14, No. 1, 1976, pp. 57-63. 
[25] McCroskey, W., "The phenomenon of dynamic stall," NASA TM 81264, NASA, 1981.

[26] Carr, L. and McCroskey, W., "A Review of Recent Advances in Computational and Experimental Analysis of Dynamic Stall," International Union of theoretical and Applied Mechanics on Fluid Dynamics at High Angle of Attack, Sept. 1992.

[27] Ekaterinaris, J. A. and Platzer, M. F., "Computational prediction of airfoil dynamic stall," Progress in Aerospace Sciences, Vol. 33, No. 11-12, 1998, pp. 759 846.

[28] Johnson, W. and Ham, N., "ON the Mechanism of Dynamic Stall," Journal of the American Helicopter Society, Vol. 17, No. 4, 1972, pp. 36-45.

[29] Spentzos, A., Badcock, K., Barakos, G., Richards, B., Wernert, P., Schreck, S., and Raffle, M., "Investigation of three-dimensional dynamic stall using computational fluid dynamics," AIAA Journal, Vol. 43, No. 5, 2005, pp. 1023-1033.

[30] Sahoo, D., Bowersox, R. D. W., and Goss, L., "Experimental investigation of the leading-edge flow of a dynamically pitching airfoil," 46th AIAA Aerospace Sciences Meeting, No. AIAA 2008-651, 2008.

[31] Vannelli, R., Particle Image Velocimetry Near The Leading Edge Of A Sikorsky SSC-A09 Wing During Dynamic Stall, Master's thesis, Texas A\&M University, 2011.

[32] Sahoo, D., Experimental Analysis of the Vorticity and Turbulent Flow Dynamics of a Pitching Airfoil at Realistic Flight (Helicopter) Conditions, Ph.D. thesis, Texas A\&M University, 2008. 
[33] Menon, R. and Wing, L., "Key Considerations in the selection of seed particles for LDV Measurements," Fourth International Conference on Laser Anemometry, 1991.

[34] Samimy, M. and Lele, S. K., "Motion of particles with inertia in a compressible free shear layer," Physics of Fluids, Vol. 3, No. 8, 1991, pp. 1915-1923.

[35] Benedict, L. H. and Gould, R. D., "Towards better uncertainty estimates for turbulence statistics," Experiments in Fluids, Vol. 22, No. 2, 1996, pp. 129-136.

[36] Hussain, A. K. M. F. and Reynolds, W. C., "The mechanics of an organized wave in turbulent shear flow," Journal of Fluid Mechanics, Vol. 41, 1970, pp. 241-258.

[37] Huang, N., Wu, M., Qu, W., Long, S. R., Shen, S., and Zhang, J., "Applications of Hilbert-Huang transform to non-stationary financial time series analysis," Applied Stochastic Models in Business and Industry, Vol. 19, No. 3, 2003, pp. 245-268.

[38] Haller, G., "Exact theory of unsteady separation for two-dimensional flows," Journal of Fluid Mechanics, Vol. 512, 2004, pp. 257-311.

[39] Lyn, D. A. and Rodi, W., "The flapping shear layer formed by flow separation from the forward corner of a square cylinder," Journal of Fluid Mechanics, Vol. 267, 1994, pp. 353-376. 